\title{
Understanding the physical properties of hybrid perovskites for photovoltaic applications
}

\author{
Jinsong Huang *1,2, Yongbo Yuan ${ }^{1,3}$, Yuchuan Shao ${ }^{1}$ and Yanfa Yan ${ }^{4}$
}

Abstract | New photovoltaic materials have been searched for in the past decades for clean and renewable solar energy conversion with an objective of reducing the levelized cost of electricity (that is, the unit price of electricity over the course of the device lifetime). An emerging family of semiconductor materials - organic-inorganic halide perovskites (OIHPs) - are the focus of the photovoltaic research community owing to their use of low cost, nature-abundant raw materials, low-temperature and scalable solution fabrication processes, and, in particular, the very high power conversion efficiencies that have been achieved within the short time of their development. In this Review, we summarize and critically assess the most recent advances in understanding the physical properties of both 3D and low-dimensional OIHPs that favour a small open-circuit voltage deficit and high power conversion efficiency. Several prominent topics in this field on the unique properties of OIHPs are surveyed, including defect physics, ferroelectricity, exciton dissociation processes, carrier recombination lifetime and photon recycling.

The impact of ion migration on solar cell efficiency and stability are also critically analysed.

Finally, we discuss the remaining challenges in the commercialization of OIHP photovoltaics.

'Department of Mechanical and Materials Engineering, University of NebraskaLincoln, Lincoln, Nebraska 68588-0656, USA.

2Department of Applied Physical Sciences, University of North Carolina, Chapel Hill, North Carolina 27599, USA. ${ }^{3}$ Hunan Key Laboratory of Super Microstructure and Ultrafast Process, School of Physics and Electronics, Central South University, Changsha, Hunan 410083, P. R. China.

${ }^{4}$ Department of Physics and Astronomy, and Wright Center for Photovoltaics Innovation and

Commercialization, University of Toledo, Toledo, Ohio 43606, USA.

Correspondence to J.H. jhuang2@unl.edu

doi:10.1038/natrevmats.2017.42 Published online 4 Jul 2017
The past few years witnessed the extremely rapid development of organic-inorganic halide perovskite (OIHP) photovoltaics. The power conversion efficiency (PCE) of small-size (typically $<15 \mathrm{~mm}^{2}$ ) perovskite solar cells has rocketed from $3.8 \%$ to a certified $22.1 \%$, approaching that of commercialized rivals, such as polycrystalline silicon, cadmium telluride (CdTe) and copper indium gallium (di)selenide (CIGS) photovoltaics. The parameters that determine the PCE are described in BOX 1. Benefiting from their ionic nature, the semiconductors in the OIHP material family have good solubility, which enables versatile low-temperature solution processes (including spin-coating, dip-coating, spray-coating, ink-jet printing, doctor-blading and slot-die coating), many of which are scalable and are thus compatible with roll-to-roll large-scale manufacturing processes. By contrast, these OIHP materials can also be deposited as films by vacuum-based techniques, such as thermal evaporation, which have yielded devices with similar performance to those made by solution processes. If the films are properly processed to eliminate the current leakage spots, OIHP solar cells can also be superior to other solution-processed solar cells, such as organic photovoltaic (OPVs), because they can be scaled up to a reasonably large area of $1 \mathrm{~cm}^{2}$ or larger without a significant reduction of the PCE. The PCEs of large-area perovskite devices $\left(1 \mathrm{~cm}^{2}\right)$ are currently approaching those of small area devices ${ }^{1}$. This is still challenging in OPVs because of the short charge-collection length in organic semiconductors, which is limited by their low carrier mobility, although current-leaking-free OPVs can be easily formed by increasing the thickness of the photoactive layer and charge transport layer. The last piece of the puzzle is to find out whether large-area devices fabricated using scalable fabrication process can retain their high efficiency.

Despite the incredible progress in efficiency enhancement for perovskite solar cells, there are still many obstacles on their way towards commercialization. Low thermal stability ${ }^{2,3}$, low resistance to moisture ${ }^{4,5}$ and the toxicity of lead are still clouds on the horizon with the current workhorse OIHP materials. New materials need to be designed and developed to overcome these obstacles; however, numerous attempts have resulted in very limited success. Direct analogies to current perovskite materials have been made to design new materials, but none of these have shown promising device performance. Moreover, clear design rules for the next generation of materials are lacking. Thus, it is necessary to summarize the findings of the perovskite photovoltaic 
community for what makes lead halide perovskites exceptional for solar cell applications. The understanding is crucial for the further improvement of perovskite solar cells and for the development of next-generation photovoltaic materials beyond OIHPs. In this Review, on the basis of the most fundamental material and physical properties, we critically assess the advances in understanding the unique fundamental optoelectronic properties of OIHP materials that explain why they work so well for solar cells, and discuss several unique characteristics, such as the influence of ion migration on the device performance.

\section{Box 1 | Open-circuit voltage deficit in OIHP solar cells}

The power conversion efficiency (PCE) of a solar cell is determined by the product of short-circuit current density $\left(U_{\text {SC }}\right)$, open-circuit voltage $\left(V_{\text {OC }}\right)$, and the fill factor (FF). One significant feature of high-efficiency organic-inorganic halide perovskite (OIHP) solar cells is the small $V_{\text {OC }}$ deficit, which is defined by $E_{g} / q-V_{O C}$, where $E_{g}$ is the optical bandgap and $q$ is elemental charge. As shown in the figure, the $V_{\text {OC }}$ deficit in the best OlHP solar cells $(\sim 0.37 \mathrm{eV})$ is much smaller than that of organic $(>0.6 \mathrm{eV})$ and quantum dot solar cells, and is even smaller than that of inorganic c-Si $(0.44 \mathrm{eV})$ and close to that of copper indium gallium (di)selenide (CIGS) solar cells $(0.38 \mathrm{eV})$. It is noted that the $V_{\text {OC }}$ deficits are compared among record efficiency solar cells, which have relatively large $J_{\mathrm{SC}}$ and $\mathrm{FF}$, indicating that charge collection is not limited by the photoactive layer or the interfaces in these devices. Although a relatively small $V_{\mathrm{OC}}$ deficit has also been recently observed in some organic solar cells, these devices generally have much smaller $J_{\mathrm{SC}}$ and FF. Because most OIHP solar cells are fabricated by low-temperature solution processes, such a small $V_{\text {OC }}$ deficit is commendable. There has been much research undertaken to understand the origin of the high $V_{\text {OC }}$ of perovskite solar cells; this constitutes a major proportion of this Review.

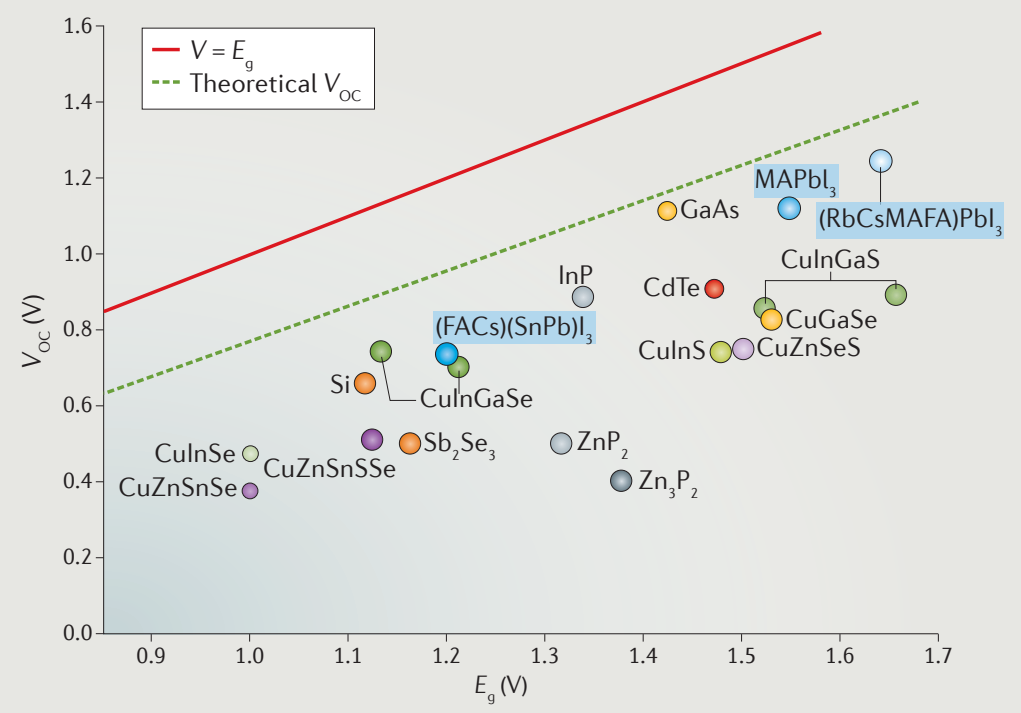

The maximum $V_{\text {OC }}$ of a solar cell is set by the Shockley-Queisser (SQ) limit, $V_{\text {OC }}^{\text {SO }}$ (REF. 152). The $V_{\text {OC }}$ loss with respect to the bandgap $\left(E_{g} / q-V_{O C}^{S O}\right)$ increases by only about $6 \mathrm{meV}$ as the bandgap increases by $100 \mathrm{meV}$. Therefore, $E_{g} / q-V_{O C}{ }^{\mathrm{SO}}$ does not vary appreciably for typically used photovoltaic materials. For c-Si with $E_{g} \approx 1.12 \mathrm{eV}$, the $E_{g} / q-V_{O C}^{S O}$ is about $250 \mathrm{meV}$ (REF. 153) and for methylammonium lead iodide $\left(\mathrm{MAPbl}_{3}\right.$ ) with $E_{9} \approx 1.55 \mathrm{eV}$, the $E_{g} / q-V_{\mathrm{OC}}^{\text {SO }}$ is about $280 \mathrm{meV}$ (REF. 154). The $V_{\mathrm{OC}}$ in a solar cell is determined by the non-radiative recombination of photogenerated charges, which reduces the splitting of the quasi-Fermi level of electrons and holes $\left(\Delta V_{\mathrm{OC}}^{\text {non-rad }}=-k T \ln \left(\mathrm{EQE}_{\mathrm{EL}}\right)\right)$, where $E Q E_{\mathrm{EL}}$ is the external quantum efficiency of electrical luminescence ${ }^{155}, k$ is the Boltzmann constant and $T$ is temperature. Therefore, reducing the defect density in solar cells is the main strategy to reduce $\Delta V_{\mathrm{OC}}^{\text {non-rad. }}$ An OIHP solar cell with a record $\mathrm{EQE}_{\mathrm{EL}}$ of $0.5 \%{ }^{156}$ resulted in a $\Delta \mathrm{V}_{\mathrm{OC}}^{\text {non-rad }}$ of just $\sim 0.13 \mathrm{~V}$.

\section{Charge recombination in perovskites}

Light absorption and charge generation. A good photovoltaic material should have a large absorption coefficient. Besides efficient light harvesting, a large absorption coefficient also contributes to a large open-circuit voltage $\left(V_{\text {OC }}\right)$, because the material would require only a thin active layer to fully absorb light with energy above the bandgap, which can reduce the charge recombinationinduced saturated dark current. This is described by the Shockley-Queisser model of the relationship between the film thickness and $V_{\text {OC }}$ (REF. 6):

$$
V_{\mathrm{OC}}=\frac{k T}{q} \ln \left(\frac{J_{\mathrm{SC}}}{J_{0}}\right)=\frac{k T}{q} \ln \left(\frac{J_{\mathrm{SC}} N_{\mathrm{D}} \tau_{\mathrm{eff}}}{q n_{\mathrm{i}}^{2} d}\right)
$$

where $k$ is the Boltzmann constant, $T$ is the temperature, $q$ is elemental charge, $J_{\mathrm{SC}}$ is short-circuit current density, $J_{0}$ is initial current density, $N_{\mathrm{D}}$ is the doping concentration, $\tau_{\text {eff }}$ is the effective carrier recombination lifetime, $n_{\mathrm{i}}$ is the intrinsic carrier concentration and $d$ is the thickness of the light absorber. Hybrid perovskites are among the most efficient photovoltaic absorbers; for example, the absorption coefficient of methylammonium lead iodide $\left(\mathrm{MAPbI}_{3}\right)\left(>3.0 \times 10^{4} \mathrm{~cm}^{-1}\right.$ in the visible light region) is more than one order of magnitude larger than that of silicon. As a result, the thickness of most reported high-efficiency OIHP solar cells is in the range of 0.3-0.6 $\mu \mathrm{m}$ (REFS 7,8), whereas crystalline silicon solar cells are usually about $300 \mu \mathrm{m}$ thick ${ }^{9}$. State-of-the-art scalable coating technologies, such as blade-coating, can deposit perovskite films with sub-micrometre thickness. A thin photoactive layer also requires less material and is thus lower in cost. The strong absorption of $\mathrm{MAPbI}_{3}$ has been attributed to its direct bandgap and $\mathrm{Pb} 6 p-\mathrm{I} 5 p$ transitions ${ }^{10-12}$.

A major form of $V_{\mathrm{OC}}$ loss in solution-processed OPVs is the loss of energy to separate light-generated Frenkel excitons, which have large binding energies in the range of 0.2-1.0 eV (REF. 13). As a consequence, a donor/acceptor heterojunction interface with a large energy offset $(>0.3 \mathrm{eV})$ is necessary in OPVs to provide the internal electrochemical driving force for exciton dissociation. The exciton binding energy $\left(E_{\mathrm{B}}\right)$ is the energy required to dissociate excitons into free charge carriers before they can be collected. For photovoltaic applications, a small $E_{\mathrm{B}}$ is favourable to minimize the energy loss. For example, many inorganic semiconductors exhibit easy charge separation owing to their small binding energies ( $\mathrm{Si}$ : $\sim 15 \mathrm{meV}$ (REF. 14); GaAs: $\sim 4 \mathrm{mV}$ (REF. 15)), because the thermal energy at room temperature is sufficiently large to dissociate excitons. The hybrid nature of $\mathrm{MAPbI}_{3}$ makes one wonder whether its behaviour, with respect to free charge generation, is similar to that of inorganic or organics materials. To answer this question, the $E_{\mathrm{B}}$ in $\mathrm{MAPbI}_{3}$ has been intensely studied by methods such as dielectric constant measurement ${ }^{16}$, optical spectroscopy $^{17}$ and magnetoabsorption spectroscopy ${ }^{18}$, with reported values varying from just $2 \mathrm{meV}$ (determined using static dielectric constant, $\varepsilon_{\text {static }}^{\prime}=70$ ) to $75 \mathrm{meV}$ (REFS 16-21) (FIG. 1a). Nevertheless, according to numerical simulations, the free charge dominates the photogenerated species under solar cell operating conditions, even 


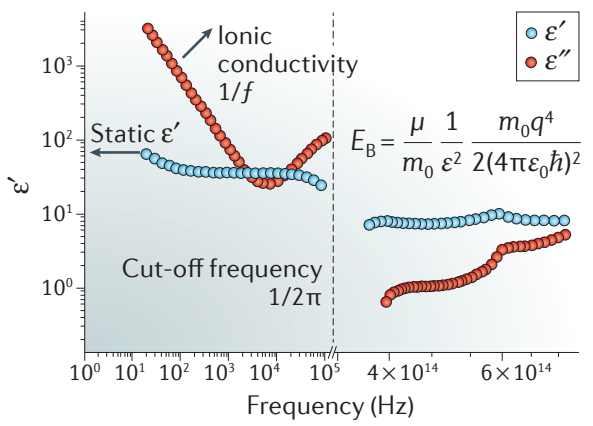

d

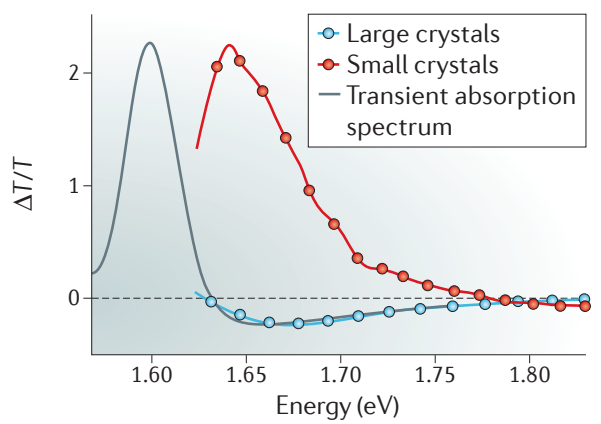

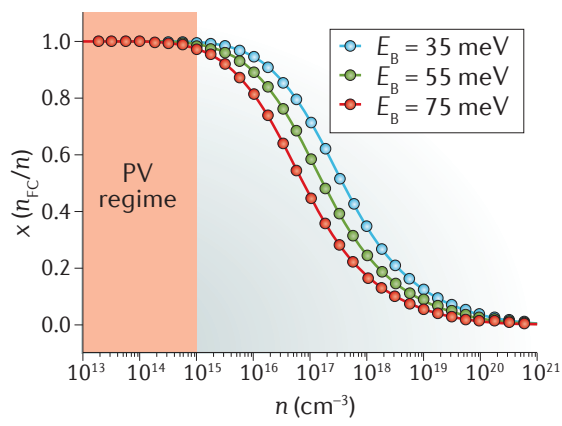

e

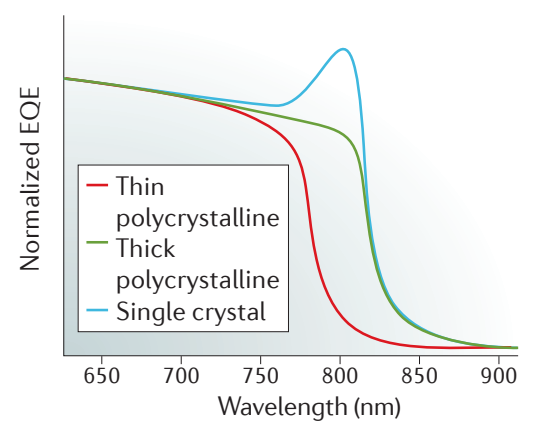

C

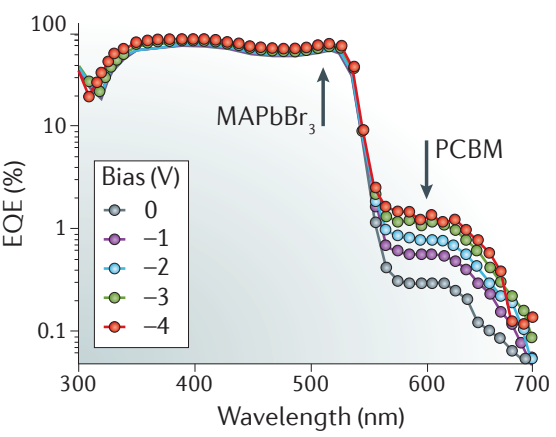

f

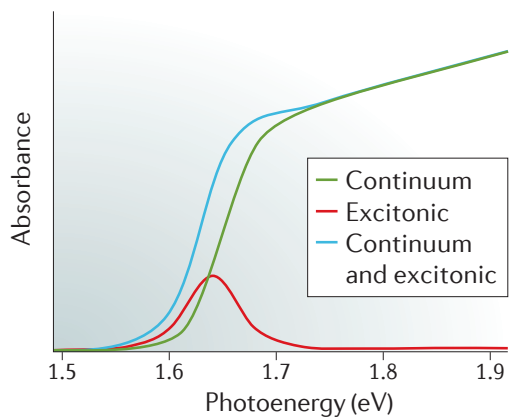

Figure 1 | Exciton or free charge generation under illumination in OIHPs? a| Real $\left(\varepsilon^{\prime}\right)$ and imaginary $\left(\varepsilon^{\prime \prime}\right)$ parts of the dielectric constants of methylammonium lead iodide $\left(\mathrm{MAPbl}_{3}\right)$. The exciton binding energy is calculated using the Wannier equation as depicted in the figure, where $\mu$ is the exciton effective mass, $m_{0}$ is the electron mass, $\varepsilon_{0}$ is the vacuum permittivity, $q$ is the elementary charge and $\hbar$ is the reduced Planck constant. $\mathbf{b} \mid$ Simulation of the free charge fraction over the total excitation density $\left(x=n_{\mathrm{FC}} / n\right)$ at thermal equilibrium, where $n_{\mathrm{FC}}$ and $n$ are the density of free charges and total excitation, respectively. The PV region is the photovoltaic region, $c$ | External quantum efficiency (EQE) spectra of $\mathrm{MAPbBr}_{3}$ solar cells with a phenyl- $\mathrm{C}_{61}$-butyric acid methyl ester (PCBM) layer under increasing reverse bias from 0 to $-4 \mathrm{~V}$; EQE signals between 560 and $700 \mathrm{~nm}$ are attributed to the PCBM. d | Transient absorption spectra at room temperature (at $1 \mathrm{ps}$ pumpprobe delay) of samples with small $(<200 \mathrm{~nm})$ and large crystals $(\sim 1 \mu \mathrm{m})$. e | Normalized EQEs of $3 \mathrm{~mm}$ - and $200 \mathrm{~mm}$-thick

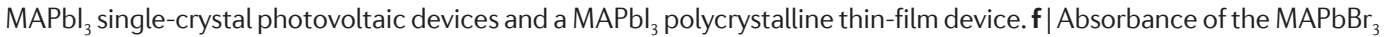
perovskite single crystal (red line) obtained from ellipsometry measurements. $f$, frequency; OIHP, organic-inorganic hybrid perovskite. Part $\mathbf{a}$ is adapted with permission from REF. 16, Macmillan Publishers Limited. Part b is adapted with permission from REF. 17, Macmillan Publishers Limited. Part $\mathbf{c}$ is adapted with permission from REF. 22, Wiley-VCH. Part d is adapted with permission from REF. 23, Macmillan Publishers Limited. Part $\mathbf{e}$ is adapted with permission from REF. 24, AAAS. Part $\mathbf{f}$ is adapted with permission from REF. 27, Macmillan Publishers Limited.

with a high $E_{\mathrm{B}}$ of $75 \mathrm{meV}$ (REF. 17) (FIG. 1b). These results suggest that $\mathrm{MAPbI}_{3}$ can be classified as a non-excitonic material in photovoltaic applications. Although different exciton binding energies have been obtained using different techniques, a consensus has been reached that the direct generation of free charges dominates the conversion process from photons to photocurrent in OIHP photovoltaic devices. The non-excitonic character enables the fabrication of planar homojunction or heterojunction thin-film solar cell structures for perovskite solar cells. Similar to the case for $\mathrm{MAPbI}_{3}, \mathrm{MAPbBr}_{3}$ also exhibits non-excitonic behaviour with a slightly higher $E_{\mathrm{B}}$ of $80 \mathrm{meV}$. Electric field-dependent external quantum efficiency (EQE) measurements were conducted for $\mathrm{MAPbBr}_{3}$ and organic excitonic material phenyl- $\mathrm{C}_{61}$-butyric acid methyl ester (PCBM) in the same device $^{22}$. Excitons in $\mathrm{MAPbBr}_{3}$ attained a maximum dissociation without the assistance of an external field, in sharp contrast to PCBM (FIG. 1c). The free-charge generation under illumination in relatively narrow bandgap perovskites, such as $\mathrm{MAPbI}_{3}$, contributes to the small $V_{\mathrm{OC}}$ loss in OIHP solar cells.

The $E_{\mathrm{B}}$ in perovskites is also found to be sensitive to their microstructure ${ }^{23}$ (FIG. 1d). Using transient absorption spectroscopy, the exciton features in $\mathrm{MAPbI}_{3}$ were found in samples with large grain sizes of $\sim 1 \mu \mathrm{m}$. In perovskite thin films with small grains, disorders and variations of electrostatic potential decrease the $E_{\mathrm{B}}$ and suppress exciton formation, which explains why the strong excitonic peak of $\mathrm{MAPbI}_{3}$ can only be observed in large single crystals $^{24}$ (FIG. 1e). A very recent study revealed strong heterogeneity in terms of free carrier or exciton generation in perovskite grains using transient absorption microscopy with a spatial resolution of $150 \mathrm{~nm}$. It was found that both excitons and free charges are generated in single perovskite grains but are spatially separated. Excitons were more likely generated at locations with higher crystallinity, and free charges were found at the centre of grains with many small domains ${ }^{25}$. The definition of 'grain' should be unified, because each grain actually comprises 
many grains owing to the special grain-growth process used in this study.

Excitons have been found to contribute to light absorption, particularly those that are close to the band edge of $\mathrm{MAPbI}_{3}$. The absorption spectrum of $\mathrm{MAPbI}_{3}$ has been decoupled to the excitonic and continuum transitions, resulting in a direct bandgap of $1.64 \mathrm{eV}$ for $\mathrm{MAPbI}_{3}$ (REFS 26,27) (FIG. 1f), which agrees with a previous study on the photoluminescence of a single crystal in which exciton emission was inhibited ${ }^{24}$. The contribution of excitonic transitions to the absorption spectrum could probably also contribute to the small Urbach energy of perovskite materials. In large perovskite crystals, the absorption spectra extend by $50 \mathrm{~nm}$ into the longer wavelength range. In devices based on thick crystals, photogenerated charges can still be efficiently collected across the very thick crystals, contributing to a larger photocurrent. Thus, tuning the crystal size can narrow down the effective bandgap without changing the composition of the perovskite. The origin of the optical transitions that result in extension of the absorption spectrum and narrowing of the bandgap is unknown. We speculate that they might be related to bound excitons, impurity-related tail states or the indirect absorption of the perovskite.

In addition to composition and microstructure, the dimensionality of the crystal structure also influences exciton binding energies. Recently, 2D hybrid perovskites, consisting of lead iodide octahedral layers intercalated with large organic cations as spacers, have gained considerable research interest because of the prospect of enhancing moisture stability ${ }^{28-31}$. Two-dimensional perovskites are essentially quantum wells: the charge carriers are mainly confined in the lead iodide octahedral planes because the organic layers have a larger bandgap and smaller dielectric constant. However, 2D perovskites suffer from having much larger exciton binding energies owing to spatial quantum confinement ${ }^{32}$. Exciton binding energies of 380 and $270 \mathrm{meV}$ have been reported in $(\mathrm{BA})_{2}(\mathrm{MA})_{n-1} \mathrm{~Pb}_{n} \mathrm{I}_{3 n+1}$ exfoliated single crystals with $n=1$ and 2, respectively, with the exciton binding quickly approaching that of the $3 \mathrm{D}$ structure with increasing $n$

Table 1 | Summary of $k_{1}$ and $k_{2}$ from different material measurements

$\begin{array}{llllr}\text { Material } & \text { Measurement } & \mathbf{k}_{\mathbf{1}}\left(\mathbf{s}^{-1}\right) & \mathbf{k}_{\mathbf{2}}\left(\mathbf{c m}^{\mathbf{3}} \mathbf{s}^{-1}\right) & \text { Refs } \\ \mathrm{MAPbl}_{3} & \text { TRPL } & 1.5 \times 10^{-5} & 6.0 \times 10^{-11} & 37 \\ \mathrm{MAPbl}_{3} & \text { Transient spectroscopy } & 1.4 \times 10^{-5} & 9.2 \times 10^{-10} & 38 \\ \mathrm{MAPbl}_{3-x} \mathrm{Cl}_{x} & \text { Transient spectroscopy } & 5 \times 10^{-6} & 9.0 \times 10^{-11} & 38 \\ \mathrm{MAPbl}_{3-x} \mathrm{Cl}_{x} & \text { Transient spectroscopy } & 1.2 \times 10^{-5} & 1.1 \times 10^{-10} & 39 \\ \mathrm{MAPbl}_{3} & \text { TRPL } & 1.8 \times 10^{-5} & 1.7 \times 10^{-10} & 40 \\ \mathrm{MAPbl}_{3-x} \mathrm{Cl}_{x} & \text { TRPL } & 3.2 \times 10^{-6} & 1.3 \times 10^{-10} & 41 \\ \mathrm{MAPbl}_{3} & \text { Transient spectroscopy } & & 2.3 \times 10^{-9} & 42 \\ \mathrm{MAPbl}_{3-x} \mathrm{Cl}_{x} & \text { Transient spectroscopy } & & 7.4 \times 10^{-11} & 43 \\ \mathrm{MAPbl}_{3} & \text { TRMC } & 7 \times 10^{-6} & 1.3 \times 10^{-9} & 44 \\ \mathrm{MAPbl}_{3} & \text { TRMC } & & 8.0 \times 10^{-12} & 45\end{array}$

$k_{1}$, monomolecular charge recombination rate constant; $k_{2}$, bimolecular charge recombination rate constant; TRPL, time-resolved photoluminescence; TRMC, time-resolved microwave conductance.
(REFS 33-35). Nevertheless, large exciton binding energies have not prevented 2D perovskites from being applied in stable and high-efficiency (PCE $>12 \%$ ) solar cells ${ }^{33}$. An important question arising in the community is how excitons in $2 \mathrm{D}$ perovskites can be efficiently dissociated into free carriers. In a recent study, it was proposed that excitons in 2D perovskites dissociate into long-lived free carriers with the help of the edge states ${ }^{33}$. The nature of the proposed edge states is not yet clear. However, in this study, the edge states were only observed in $2 \mathrm{D}$ perovskites with $n \geq 3$, in which the $E_{\mathrm{B}}$ is much smaller. Therefore, despite solid evidence for the presence of edge states in $2 \mathrm{D}$ perovskites, their contribution to the efficient conversion of excitons to free charge carriers is not yet clear. More in-depth investigations of the charge-carrier dynamics in $2 \mathrm{D}$ perovskites, especially for low values of $n$, are urgently needed to enable further optoelectronic applications.

Charge recombination rate. A longer carrier recombination lifetime is needed for larger $V_{\mathrm{OC}}$ because it enables a higher carrier concentration (or a larger quasi-Fermi level splitting) for solar cells under illumination. The charge recombination in a semiconductor material can be described by the following equation ${ }^{36}$ :

$$
\frac{\mathrm{d} n}{\mathrm{~d} t}=G-k_{1} n-k_{2} n^{2}-k_{3} n^{3}
$$

where $G$ is the charge generation rate, $k_{1}$ is the monomolecular charge recombination rate constant, $k_{2}$ is the bimolecular charge recombination rate constant and $k_{3}$ is the Auger charge recombination rate. The Auger charge recombination is generally weak compared with other recombination channels at normal solar cell operating conditions (that is, under 1 sun illumination with a carrier concentration of $10^{15}-10^{16} \mathrm{~cm}^{-3}$ ). Different techniques, including time-resolved photoluminescence (TRPL), transient spectroscopy and time-resolved microwave conductance (TRMC), have been used to determine the values of $k_{1}$ and $k_{2}$; the results are summarized in TABLE 1 (REFS 37-45). Bimolecular (or second-order) recombination rate constants are typically reported to be in the range of $10^{-11}-10^{-9} \mathrm{~cm}^{3} \mathrm{~s}^{-1}$ at room temperature for both polycrystalline films and single crystals, which is about 3-5 orders of magnitude lower than what is predicted by the Langevin model ${ }^{46}$. The reported monomolecular (or first-order) recombination rate constant in most polycrystalline $\mathrm{MAPbI}_{3}$ films is between $10^{-7}$ and $10^{-6} \mathrm{~s}^{-1}$, but it can reach $10^{-5} \mathrm{~s}^{-1}$ or slower (that is, a photoluminescence lifetime of $10 \mu \mathrm{s}$ or longer) in passivated polycrystalline films and single crystals $^{24,47,48}$. The very weak bimolecular charge recombination makes monomolecular charge recombination the dominant recombination process in most polycrystalline perovskite films under low excitation intensity.

An important question that needs particular attention is how to accurately measure the first-order charge recombination lifetime. TRPL has been frequently applied to evaluate the quality of the perovskite films, but the measured lifetime does not correspond to the charge recombination lifetime in the device under open-circuit conditions. The photoluminescence lifetime 
of surface-passivated perovskite films can be as long as several microseconds ${ }^{49}$, whereas the charge recombination lifetime measured in solar cells under open-circuit conditions is only in the sub-microsecond range, even for optimized devices with PCEs of $>20 \%$. This is because the charge transport layers in real devices can accelerate charge dissociation (that is, the separation of photogenerated electrons and holes) such that the radiative charge recombination is too weak to be monitored in TRPL measurements. This suggests the existence of an additional carrier recombination channel in real devices, the understanding of which could help further enhance the device efficiency. A recent study of the single-wall carbon nanotube (SWCNT)/perovskite interface revealed a carrier lifetime of several hundred microseconds for charge-separated states in thin films deposited on glass without electrodes ${ }^{50}$. Therefore, the TRPL lifetime of perovskite thin films should not be considered as a device parameter, although it can be a parameter to evaluate the defect density and suitability of the material for solar cells; this is owing to the commonly observed correlation between the photoluminescence lifetime and device efficiency.

Excellent progress has been made in understanding the ultralong charge carrier recombination lifetime in hybrid perovskites. First-principles calculations predict that the Rashba spin-orbit coupling effect can change the positions of the conduction band minimum (CBM) and valence band maximum (VBM) in $k$ space $^{51}$, leading to a reduced radiative recombination rate. As a result, OIHPs exhibit similar radiative recombination behaviour to indirect semiconductors ${ }^{51}$. Experimental verification or disproval of this hypothesis will be interesting. It is not surprising that single-crystal perovskites can have long photoluminescence lifetimes (above $10 \mu \mathrm{s}$ ), because a similar recombination lifetime was observed in very intrinsic semiconductor GaAs crystals with doping concentrations as low as those of hybrid perovskites $^{52}$. Nevertheless, it is very surprising that the recombination lifetime of polycrystalline films formed by a low-temperature solution process can be as long as hundreds of nanoseconds, despite their grain size being similar to those of CdTe or CIGS thin films. The easy crystallization due to the ionic nature of inorganic salt precursors should contribute to the low point defect density in perovskite materials. An in situ X-ray scattering study revealed a low crystallization activation energy barrier of $56.6-97.3 \mathrm{~kJ} \mathrm{~mol}^{-1}$, depending on the different lead salts used as precursors ${ }^{53}$. By contrast, the nucleation and crystallization activation energy of amorphous silicon (a-Si) can be as high as $\sim 470$ and $280 \mathrm{~kJ} \mathrm{~mol}^{-1}$, respectively ${ }^{54}$. Benefiting from such a low crystallization energy barrier, perovskite thin films with excellent crystallinity and low defect density have been fabricated by a range of techniques, including low-temperature solution processes, which is an advantage for commercialization considering the low processing cost. Moreover, the important concept of defect tolerance for hybrid perovskite materials was proposed to explain the long carrier recombination lifetime of polycrystalline films; we discuss this further in the next section ${ }^{55}$.
Most of the polycrystalline films in solar cells with efficiencies $>20 \%$ still have a film photoluminescence lifetime much shorter than $10 \mu \mathrm{s}$. This indicates that the efficiency or $V_{\text {OC }}$ of these state-of-the-art solar cells is still limited by the trap-related charge recombination in the perovskite films, in addition to the charge recombination at the perovskite/electrode interfaces.

Charge screening. Non-Langevin-type bimolecular recombination (the Langevin model proposes that the bimolecular recombination coefficient is determined by $e\left(\mu_{\mathrm{e}}+\mu_{\mathrm{h}}\right) /\left(\varepsilon \varepsilon_{0}\right)$, where $e, \mu_{\mathrm{e}}, \mu_{\mathrm{h}}$ and $\varepsilon \varepsilon_{0}$ are the electron charge, electron mobility, hole mobility and dielectric constant, respectively) is generally seen in almost all efficient solar cell technologies, such as GaAs and organic bulk heterojunction solar cells. This is particularly important for absorbers with much larger carrier mobility than organic molecules because the coefficient of Langevin-type bimolecular recombination is proportional to carrier mobilities. In general, a charge screening mechanism should reduce second-order charge recombination. The large dielectric constant of hybrid perovskite materials screens electrons and holes such that they do not feel each other's presence. Several other mechanisms have been proposed to explain the slow recombination of electrons and holes after free-charge generation in hybrid perovskites, as discussed below.

A hypothesis raised at the initial stages of perovskite solar cell research was that of a polarization field arising from the possible ferroelectricity of perovskites, which facilitates the separation of electrons and holes ${ }^{56,57}$. The perovskite structure leads one to wonder whether $\mathrm{MAPbI}_{3}$ is ferroelectric and whether the ferroelectricity, if present, contributes to the superb photovoltaic performance and the notorious photocurrent-voltage hysteresis. In both theoretical and experiment studies, it remains controversial whether $\mathrm{MAPbI}_{3}$ is ferroelectric. From a theoretical point of view, a large variation of the electric polarization from $4-38 \mu \mathrm{C} \mathrm{cm}^{-2}$ has been predicted $^{58,59}$. Nevertheless, the existence of polarization has been questioned because the antiferroelectric tetragonal structure is more stable than its ferroelectric counterpart ${ }^{60}$. It was argued that the tetragonal phase of $\mathrm{MAPbI}_{3}$ is centrosymmetric and should not display ferroelectricity at room temperature ${ }^{61-63}$. Thus, it would seem that the origin of possible polarization must rely on the ordering of dipoles of the organic cations. However, theoretical predictions and experimental evidence have recently emerged for the rapid rotation of $\mathrm{MA}^{+}$ ions in the inorganic cages at room temperature ${ }^{59,64-66}$. Experimentally, domain-like structures have been observed by piezoresponse force microscopy (PFM) imaging ${ }^{67}$; however, reliable measurements of polarization-electric field $(P-E)$ loops in $\mathrm{MAPbI}_{3}$ devices have not been reported ${ }^{4,68}$. The 'banana-shaped' $P-E$ loops obtained for many ferroelectric films could come from either back-to-back diodes ${ }^{69}$, ion migration or the leakage of current ${ }^{70}$. To prove the existence of ferroelectricity at the macroscopic level, convincing remnant polarization has to be measured below the Curie temperature. In many studies, after carefully excluding the influence 
of charge leakage by using a classical Sawyer-Tower circuit, no appreciable stable remnant polarization in $\mathrm{MAPbI}_{3}$ was detected at room temperature ${ }^{58,71,72}$ (FIG. 2a). The signals from PFM may come from artefacts such as injected charges from the excessively large applied electric field during the poling process, ion migration, grain orientation-dependent surface charge density, or even surface sensitivity to the atmosphere. Ferroelectric-like behaviour in several non-ferroelectric materials has been observed by $\mathrm{PFM}^{73,74}$. However, direct evidence, such as temperature-dependent polarization, and dielectric and structural measurements, is required to verify whether ferroelectricity exists in these materials.

Despite the difficulty in verifying the ferroelectricity of $\mathrm{MAPbI}_{3}$, a combination of microscopic and nanoscale techniques has provided solid evidence for the existence of ferroelastic domains in both $\mathrm{MAPbI}_{3}$ polycrystalline films and single crystals in the pristine state and under applied stress ${ }^{75,76}$ (FIG. 2b). A clear domain structure with $70^{\circ}$ and $109^{\circ}$ domain walls can be identified with polarized optical microscopy using bulky single crystals, and with PFM using polycrystalline thin-film samples. The presence of these domain walls can be explained by the recent confirmation of the existence of different orientations of twinning domains in the tetragonally structured $\mathrm{MAPbI}_{3}$ (FIG. 2c) with direct measurement of crystallographic orientation inside a single 'grain' (REF. 77). When the crystal was placed under stress, the change of the crystal orientation moved the domains walls, which then remained fixed after removal of the stress, similar to the polarization of ferroelectric dipoles. The motion of ferroelastic domains under applied stress in both single crystals and polycrystalline films was directly observed under polarized optical microscopy and PFM, respectively (FIG. 2d). A similar domain structure was also observed by photothermal induced resonance (PTIR), which measures changes in the crystallographic orientation-dependent thermal expansion coefficient. The twinning domain of $\mathrm{MAPbI}_{3}$ observed by PTIR did not respond to applied electrical bias, suggesting the absence of ferroelectricity in $\mathrm{MAPbI}_{3}$ (REF. 76).

Although there has been no conclusive experimental evidence presented in the literature for a 'thermodynamic' ferroelectric phase in the dark, photoferroelectricity in these materials (that is, the photoinduced reordering of organic cation dipoles, such as $\mathrm{MA}^{+}$and $\mathrm{FA}^{+}$, where FA is formamidinium) was recently predicted to be energetically favourable owing to the weakened hydrogen bonds between organic cations and inorganic cages in the excited state. The thermalization energy also assists the photoinduced organic cation dipole reordering pro$\operatorname{cess}^{78}$. This can explain the observed fivefold enhancement in piezoelectricity of $\mathrm{MAPbI}_{3}$ under illumination ${ }^{72}$ and may also be responsible for the recently reported photorestriction $^{79}$. Nevertheless, photorestriction still needs to be verified through measurements on the macroscopic scale, because nanoscale SPM measurements are subject to many artefacts. By the same token, light-driven molecular reordering also still needs to be experimentally verified, either by indirect comparison of the photopiezoelectricity of perovskites with polar or nonpolar cations (such as $\mathrm{Cs}^{+}$), or directly monitoring organic cations with infrared spectroscopy.

The effect of organic cations on charge recombination and the device $V_{\text {OC }}$ has also been investigated in hybrid perovskite materials. Without the assumption of ferroelectricity, a screen effect on the carrier recombination was suggested to be caused by the reorientation of polar organic cations ${ }^{80,81}$. In another study, $\mathrm{MA}^{+}$was replaced with $\mathrm{Cs}^{+}$in $\mathrm{MAPbBr}_{3}$; the devices constructed from both materials showed comparable $V_{\mathrm{OC}}$ and efficiency ${ }^{82}$. Efficient $\mathrm{CsPbBr}{ }_{3}$ devices were difficult to make because of the excessively large bandgap of this material at room temperature. Recent demonstration of high-efficiency $\mathrm{CsPbI}_{3}$ devices with the 'black' phase stabilized by quantum dots also showed that organic dipole cations are not necessary for reaching a large $V_{\mathrm{OC}}$ in perovskite solar cells ${ }^{83}$. The relatively lower efficiency of all inorganic perovskites is probably due to phase instability and non-optimized film morphologies. The band-edge carrier dynamics were compared in single crystals, $\mathrm{MAPbBr}_{3}, \mathrm{FAPbBr}_{3}$, and $\mathrm{CsPbBr}$, by transient spectroscopies that showed comparable carrier recombination rates regardless of the cation types ${ }^{84}$. All these studies found that the organic cations are not essential in determining the optoelectronic properties of the hybrid perovskites and device performance. In one example, although the type of organic cation had no obvious impact on the band-edge charge recombination, it did have an astonishing screening effect on the thermalization of hot carriers ${ }^{26,85}$ (FIG. 2e,f). The organic cations were found (in TRPL measurements) to be crucial for slowing the thermalization of energetic hot carriers in $\mathrm{MAPbBr}_{3}$ and $\mathrm{FAPbBr}_{3}$, whereas such a phenomenon was not observed in $\mathrm{CsPbBr}_{3}$. As shown in the lower panel of FIG. 2e, the band-edge emission (excited by low-energy $2.3 \mathrm{eV}$ photons) remains almost invariant, whereas the hot-carrier luminescence (excited by high energy $2.6 \mathrm{eV}$ photons) decays with a time constant of $t=160 \pm 10 \mathrm{ps}$. The screening effect was attributed to the liquid-like reorientational motions of organic cations. Moreover, in an earlier study of the slow thermalization of hot carriers in hybrid perovskites using transient absorption, a similar mechanism was proposed ${ }^{26}$. The long hot-carrier relaxation lifetime should result in a long hot-carrier diffusion length, given the large carrier mobility in perovskites. A quasi-ballistic transport as long as $600 \mathrm{~nm}$ within tens of picoseconds was directly visualized by imaging charge transport in $\mathrm{MAPbI}_{3}$ thin films with transient absorption microscopy ${ }^{86}$. Such long hot-carrier transport in principle will enable hot-carrier devices. Although this hot-carrier screening effect does not impact on the performance of regular perovskite solar cells, it may enable the harvesting of the hot carriers to boost the power conversion efficiency of solar cells above the Shockley-Queisser limit.

Doping in perovskite semiconductors. Doping is important for many electronic properties of semiconductors, such as conductivity and mobility. In solar cells, doping can directly change the charge carrier recombination rate $^{87}$, diffusion length ${ }^{88}$ and contact resistance ${ }^{89}$, as well 

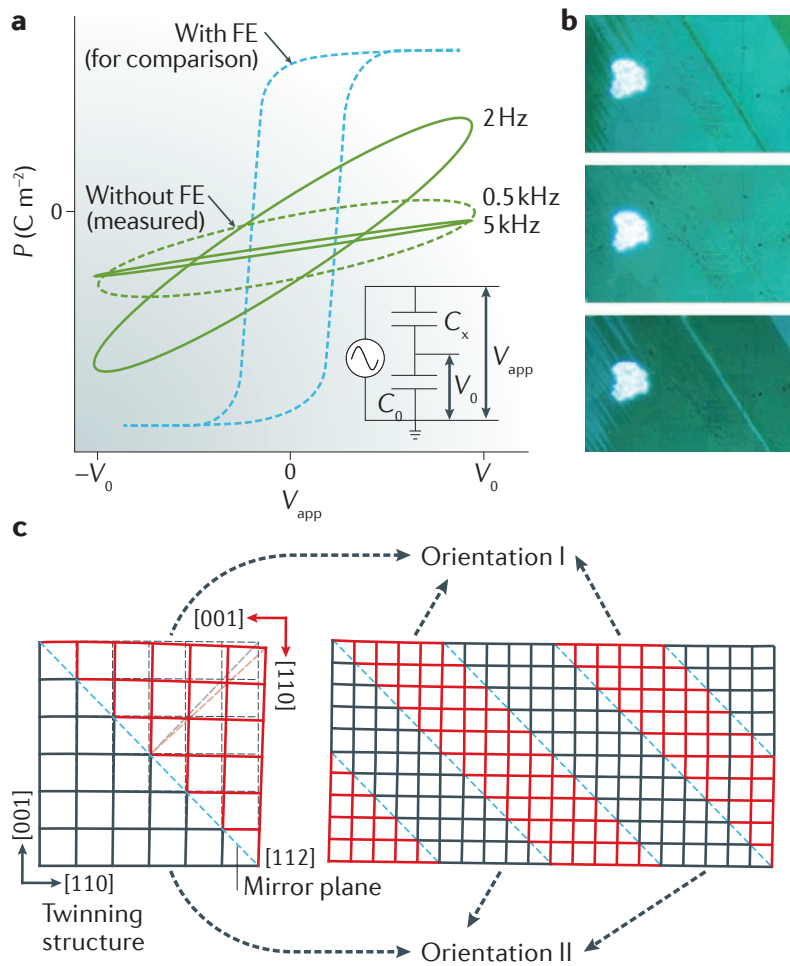
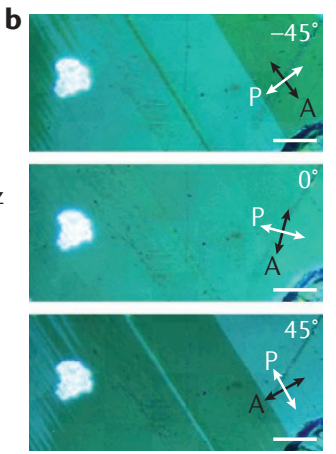

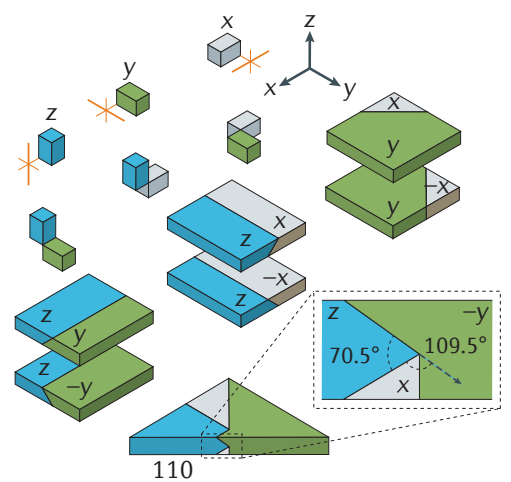

d
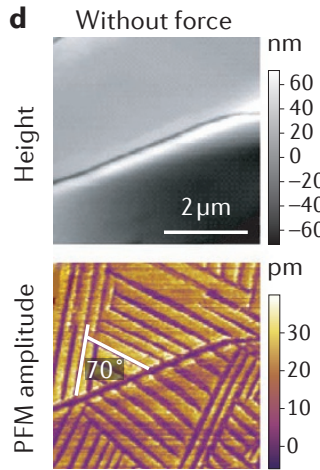

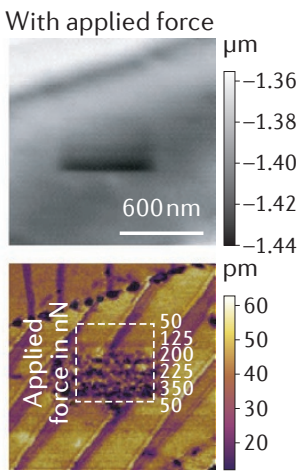

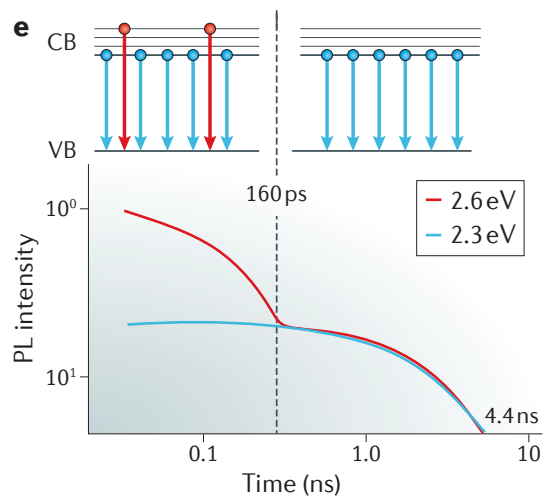

f

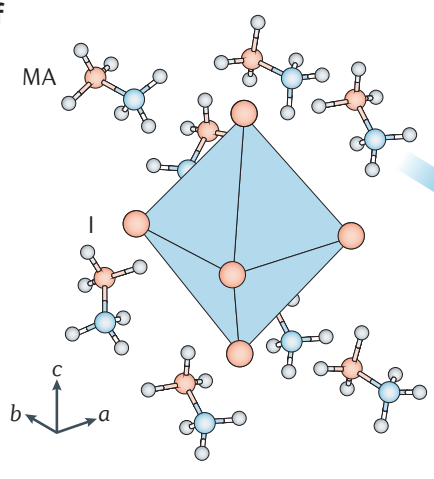

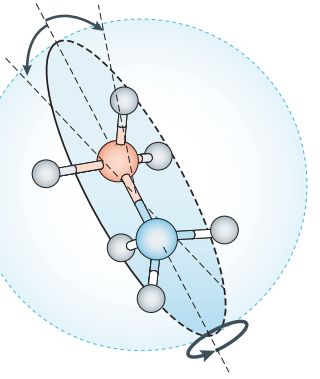

Figure 2 | Electromechanical properties of OIHPs. a | Polarization tests of large crystal methylammonium lead iodide $\left(\mathrm{MAPbl}_{3}\right)$-based complete solar cells using the Sawyer-Tower circuit in the inset, where $C_{0}$ is a linear capacitor as reference, $C_{x}$ is the measurand capacitor (that is, a capacitor with a possible ferroelectric spacing layer), $V_{\text {app }}$ is the applied bias, $V_{0}$ is the potential different on $C_{0}$, and $P$ is the polarization density. At high frequencies, the response is purely capacitive. At low frequencies, elliptic traces indicate the angular phase shift produced by resistive elements. $\mathbf{b} \mid$ Reflection mode polarized optical micrographs (crossed Nicols) showing contrast reversal of domain groups as a function of sample rotation relative to the polarizer-analyser $(\mathrm{P}-\mathrm{A})$ pair. At $0^{\circ}$ relative angle the domain groups are indistinguishable, and at $45^{\circ}$ and $-45^{\circ}$ the domain contrast is maximum (that is, $\theta_{\mathrm{e}}=90^{\circ}$ ). The white and black arrows indicate the P-A orientation. The light intensity of a bright dust particle is insensitive to the sample rotation. The scale bar is $5 \mu \mathrm{m}$ in all panels. The right panel shows the permissible domains of the $\mathrm{MAPbl}_{3}$ tetrahedral phase. At the cubic-to-tetrahedral transition, the tetrahedral unit cell can form with the $c$ axis aligned along any of the three axes $(x, y, z)$ of the parental cubic phase, leading to three possible configurations $(X, Y, Z)$. To preserve crystal integrity, the $a$ axes of different domains must coincide ${ }^{75}$, leading to six possible domain orientations, which are characterized by domain walls intercepting at $0,90^{\circ}$ or $45^{\circ}$ in the principal plains of the parental phase, and at $\sim 70^{\circ}$ and $\sim 110^{\circ}$ in the (110) plane. $\mathrm{c} \mid$ Schematic of the proposed twining structure composed of two crystal orientations in $\mathrm{MAPbl}_{3}$ crystal and the corresponding twin domains. $\mathbf{d} \mid$ Atomic force microscopy topography images and piezoresponse force microscopy (PFM) signal amplitude mapping of an as-prepared polycrystalline $\mathrm{MAPbl}_{3}$ film and a polycrystalline $\mathrm{MAPbl}_{3}$ film, in which the ferroelastic domains show clear switchable behaviour under applied force. e | Photoluminescence (PL) intensity decay kinetics at $2.3 \mathrm{eV}$ (blue line) and 2.6 eV (red line). The photoluminescence intensity at $2.6 \mathrm{eV}$ has been multiplied by a factor of $1,500 . \mathbf{f} \mid$ Crystal structure of $\mathrm{MAPbl}_{3}$ represented by an $\mathrm{ABX}_{3}$ primitive unit cell. The right panel shows the orientational disorder of MA molecules. FE, ferroelectricity; OIHP, organic-inorganic hybrid perovskite. Part $\mathbf{a}$ is adapted with permission from REF. 72, American Chemical Society. Part b is adapted with permission from REF. 16, AAAS. Part $\mathbf{c}$ is adapted with permission from REF. 16, Macmillan Publishers Limited. Part $\mathbf{d}$ is adapted with permission from REF. 76, AAAS. Part $\mathbf{e}$ is adapted with permission from REF. 85, AAAS. 
as the $V_{\text {OC }}$ (REFS 90,91). The low doping concentration in perovskites may contribute to the long carrier diffusion length because of low charge carrier scattering and recombination ${ }^{24}$, whereas a moderate doping concentration is preferred to reduce the internal resistivity of the solar cells and lead to larger $V_{\mathrm{OC}}$, as described by equation 1 (REF. 92). However, intentional extrinsic doping of hybrid perovskite materials is difficult to achieve because of the defect-tolerant nature and low activation energy for ion migration. Most polycrystalline perovskite thin films that yield highly efficient solar cells and single-crystal perovskite films are very close to being intrinsic or weakly p-type ${ }^{93,94}$, which makes the study of doping concentration and doping type difficult with Hall effect measurements ${ }^{42}$. The self-doping properties can be explained by the similar formation energies of the dominant donor and dominant acceptor ${ }^{12}$. Apparently, the self-doping level in perovskite materials is sensitive to the precursor composition and fabrication process parameters. Ultraviolet photoelectron spectroscopy (UPS) measurements showed that $\mathrm{MAPbI}_{3}$ films that formed from the pre-mixed precursor solution with more $\mathrm{PbI}_{2}$ were more $\mathrm{n}$-doped, and thermal annealing could convert the p-type perovskite to n-type by removing MAI, which may also be related to the formation of metallic lead upon perovskite decomposition. Although n-type ${ }^{95}$ doping has been observed in $\mathrm{MAPbI}_{3}$ under non-optimized film formation conditions, these heavily doped films generally do not yield high-efficiency devices. Wellcontrolled doping without reducing the carrier mobility and recombination lifetime needs to be achieved to further enhance the device $V_{\mathrm{OC}}$. It is worth mentioning that relevant characterizations of doping effects should be carried out in an inert environment. For example, a recent study into the anomalous photovoltaic effect in polycrystalline $\mathrm{MAPbBr}_{3}$ films found that the physically absorbed oxygen around grain boundaries can de-dope the perovskite films in a few minutes ${ }^{96}$.

Photon recycling. The photon recycling effect (that is, the reabsorption and re-emission by the photoactive layer itself) was proposed to contribute to the high $V_{\mathrm{OC}}$ in GaAs solar cells and thus be responsible for increasing the PCE from 25 to $29 \%$, because it allows the build-up of charge carriers in the active layer to increase the quasi-Fermi level splitting. The perovskite community is now asking the question of whether the photon recycling effect also contributes to the large $V_{\mathrm{OC}}$ in perovskite solar cells. In fact, there are many similarities between perovskites and GaAs. For example, both are direct bandgap materials and exhibit high absorption coefficients near the band edge $\left(\sim 10^{3} \mathrm{~cm}^{-1}\right)$, which gives rise to high self-absorption ratios. These properties meet some of the prerequisites for photon recycling. Recently, the first experimental evidence for the photon recycling effect in $\mathrm{MAPbI}_{3}$ polycrystalline thin films was reported, showing that charge generation was observed more than $50 \mu \mathrm{m}$ from the light-absorbing region ${ }^{97}$ (FIG. 3a-C). Meanwhile, a method was developed to determine the photon recycling efficiency in organic-inorganic hybrid single-crystal perovskites by differentiating emitted and reabsorbed photons on the basis of their polarization difference ${ }^{98}$. It was found that the photoluminescence signal from the crystals was dominated by the filtered photoluminescence emission of the surface, while emission after multiple cycles of reabsorption and re-emission is very weak (FIG. $3 d-f$ ). In these systems, the efficiencies of photon recycling were less than $0.5 \%$, showing negligible photon recycling effects under the solar cell working conditions (that is, close to 1 sun illumination intensity at room temperature). The low photon recycling efficiency may be attributed to the low internal photoluminescence quantum yield (PLQY) of the perovskite thin films and single crystals. Although a record PLQY of $93 \%$ has been reported for OIHP-based nanoparticles, this may result from the quantization effect due to spatial confinement, oscillator strength of the excitons and an increase in the binding energy. Accurately measuring the internal PLQY of perovskite thin films is difficult because of the small escape cone of emitted light inside the films. Using very rough perovskite films to enhance light outcoupling, a recent study showed that the PLQY was only as high as $60 \%{ }^{99}$, in contrast to the value of $99.7 \%$ achieved in GaAs at room temperature, despite the excitation intensity being nearly two orders of magnitude higher for the perovskite film. Another challenge for realizing photon recycling in perovskite solar cells is that the electronand hole-transport layers, which are necessary components in current cell configurations, inevitably quench the photoluminescence, resulting in a lower PLQY even under open-circuit conditions.

High electronic dimensionality. Recently, the concept of electronic dimensionality was introduced to partially explain why $\mathrm{MAPbI}_{3}$ exhibits excellent photovoltaic properties whereas other metal halide perovskite absorbers do not ${ }^{100}$. Conventionally, structural dimensionality has been used as one metric to account for photovoltaic properties and device performances of absorbers ${ }^{101-103}$. The electronic dimensionality is defined by the connectivity of the electronic orbitals that comprise the lower conduction band and the upper valence band. The electronic dimensionality is better than the conventional structural dimensionality for explaining photovoltaic properties, such as bandgaps, carrier mobilities, defect levels and device performances, of all reported halide perovskites. Although some perovskites are both structurally and electronically $3 \mathrm{D}$, some are structurally $3 \mathrm{D}$ but exhibit lower-dimensional electronic structures. For example, $\mathrm{MAPbI}_{3}$ is both structurally and electronically $3 \mathrm{D}$, whereas some double perovskites, such as the Ag- and Bi-containing halide double perovskites, are structurally $3 \mathrm{D}$ but electronically $0 \mathrm{D}$. The electronic dimensionality partially explains why $\mathrm{MAPbI}_{3}$ exhibits superior photovoltaic properties, whereas metal halide double perovskites show rather poor photovoltaic properties. Low electronic dimensionality also implies that, at a minimum, the absorber films must be grown with special crystallographic orientation to facilitate effective carrier transport. The high electronic dimensionality provides important guidance for understanding the performances of solar cells. 


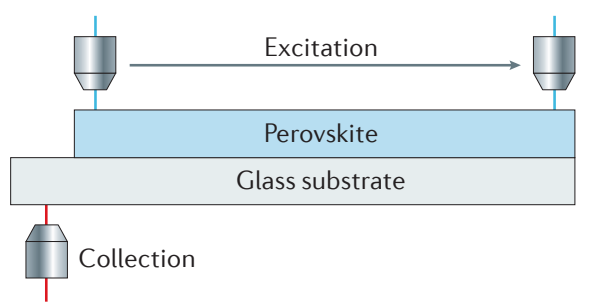

d

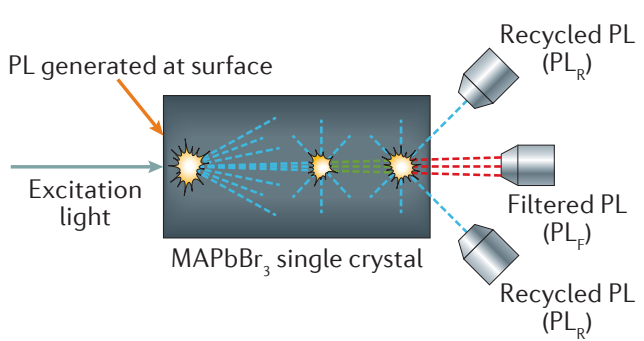

b
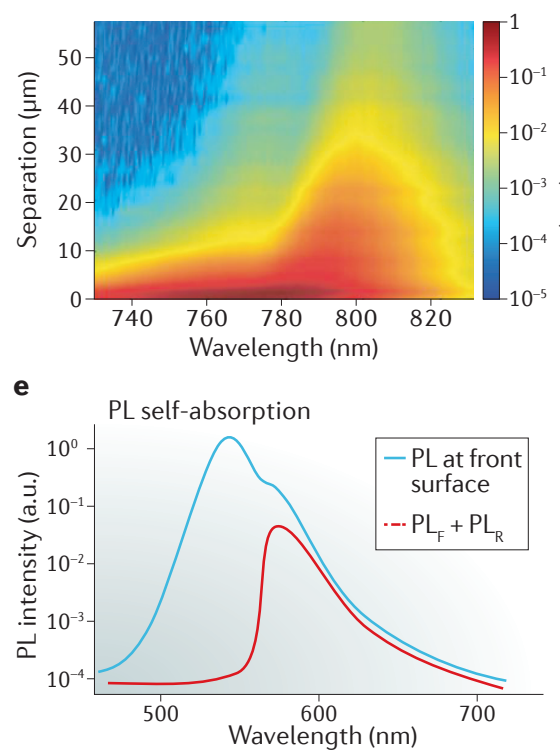

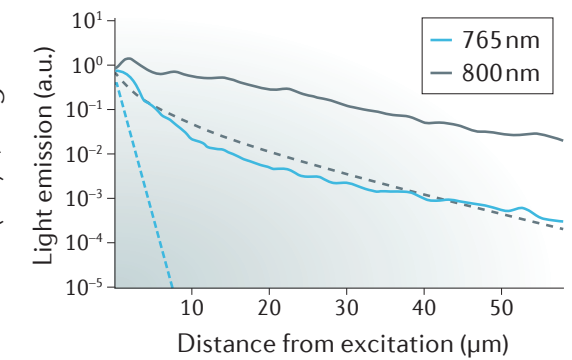

f

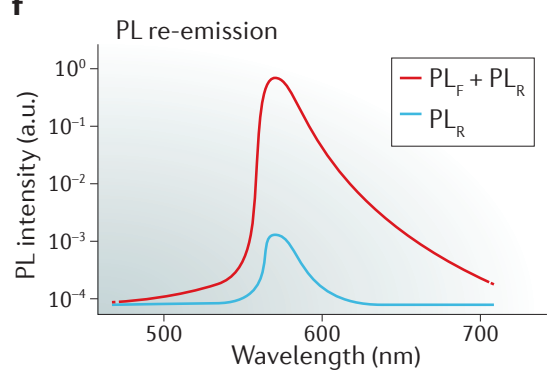

Figure 3 | Photon recycling effect in OIHPs? a | Schematic illustration of the microscope setup for photon recycling measurements. $\mathbf{b}$ | Experimentally measured light emission map for different separation distances between excitation and collection. c|Comparison between experiment (solid lines) and expected decay (dashed lines) from the BeerLambert law at 765 and $800 \mathrm{~nm}$. The experimental data are not in agreement with simple linear absorption, which suggests that additional processes, such as photon recycling, maintain substantial photon intensity at large distances. d) Schematic of the photon recycling process in perovskite single crystals. Photoluminescence (PL) excited on the surface of the single crystal is absorbed during transmission through the crystal and photons are re-emitted inside the crystal, such that the emission from the bottom of the single crystal includes both the filtered $\left(P L_{F}\right)$ and recycled $\left(P L_{R}\right)$ photoluminescence. e| Photoluminescence generated at the surface (blue curve) and the filtered and recycled photoluminescence $\left(\mathrm{PL}_{\mathrm{F}}+\mathrm{PL}_{\mathrm{R}}\right.$; red curve) spectra of a $1.3 \mathrm{~mm}$-thick methylammonium lead bromide $\left(\mathrm{MAPbBr}_{3}\right)$ single crystal. The difference between the blue curve and red curve is due to the self-absorption of $\mathrm{MAPbBr}_{3}$ single crystals. f | Combined filtered and recycled photoluminescence $\left(\mathrm{PL}_{\mathrm{F}}+\mathrm{PL} \mathrm{L}_{R}\right.$; red curve) and the recycled photoluminescence $\left(\mathrm{PL}_{\mathrm{R}}\right.$; blue curve) spectra. The re-emission ratio defined by $\mathrm{PL}_{R} / \mathrm{PL}_{\text {front-surface }}$ is very low. a.u., arbitrary units; OlHP, organicinorganic hybrid perovskite. Parts a-c are adapted with permission from REF. 97, AAAS. Parts $\mathbf{e}$ and $\mathbf{f}$ are adapted with permission from REF. 98, Macmillan Publishers Limited.

\section{Defect physics of hybrid perovskites}

Band tail of perovskites. The calculation of $V_{\mathrm{OC}}^{\mathrm{SQ}}$ assumes a perfect abrupt or step-function absorption coefficient of a light absorber (in other words, there is no absorption below the optical bandgap), which results in an overestimation of the attainable maximum $V_{\mathrm{OC}}$. In a realistic solar cell, the absorption spectrum is non-abrupt, because there is always an absorption tail due to various reasons, such as defects. Therefore, the maximum attainable $V_{\mathrm{OC}}$ - the radiative recombination limit $\left(V_{\mathrm{OC}}^{\mathrm{rad}}\right)-$ is defined by the balance of the radiative charge recombination current and the photocurrent. For any type of solar cell, $V_{\mathrm{OC}}^{\text {rad }}$ (which constitutes a path for $V_{\mathrm{OC}}$ loss due to sub-bandgap recombination) is much smaller than $V_{\mathrm{OC}}^{\mathrm{SO}}$ ). OIHP photovoltaic devices are blessed with very small $V_{\mathrm{OC}}$ loss of this type because of the small sub-bandgap absorption.

The absorption spectra of OIHPs have very steep band edges. The steepness of the absorption edge is characterized by the Urbach energy, which is defined as the slope of the exponential part of the absorption coefficient curve. The Urbach energy of polycrystalline $\mathrm{MAPbI}_{3}$ was reported to be $\sim 15 \mathrm{meV}$ at room temperature ${ }^{104,105}$, which is close to that of single crystalline silicon $(\sim 11 \mathrm{meV})$ and GaAs $(\sim 7.5 \mathrm{meV})^{104,106}$, suggesting a well-ordered microstructure and low density of deep traps in solution-processed polycrystalline $\mathrm{MAPbI}_{3}$ thin films. This is directly supported by improvements in crystallinity and phase purity in wide bandgap polycrystalline perovskite materials with $\mathrm{Cs}^{+}$incorporation, in which a reduced Urbach energy resulted in an increased device $V_{\mathrm{OC}}$ and efficiency ${ }^{107}$. The contribution of absorption from excitons should also contribute to the small Urbach energy of perovskite materials.

Point-defect tolerant hybrid perovskites. It is now generally accepted that the low density of charge traps in OIHPs contributes to the large $V_{\mathrm{OC}}$ of these solar cells; however, our understanding of the charge traps is still at an early stage. The unusually low density of deep traps derived from the absorption spectra of polycrystalline OIHP films may relate to the unusual defect physics of this family of materials. Although most OIHP devices are fabricated by low-temperature solution processes, the trap density is small compared with other polycrystalline inorganic solar cells. For example, the bulk trap density in polycrystalline perovskite thin-film-based devices is in the range of $10^{15}-10^{17} \mathrm{~cm}^{-3}$. Perovskite single crystals grown by simple solution-processed methods have an extremely low trap density of $\sim 10^{10} \mathrm{~cm}^{-3}$ (REF. 24) (FIG. 4a), 
which is comparable to the best value reported in intrinsic crystalline silicon (c-Si).

The unusual defect physics in OIHPs was recently revealed $^{55}$. The formation energies and energy levels of all possible point defects in $\mathrm{MAPbI}_{3}$ are calculated and shown in FIG. 4b,c. All point defects that can form deep traps have large formation energies and are not likely to affect the device performance, whereas most point defects that exist in the bulk of $\mathrm{MAPbI}_{3}$ either have energy levels in the conduction or valance bands or form relatively shallow traps. The defect-tolerant electronic properties of the OIHPs have been confirmed in other studies. For example, a superior synthesis of $\mathrm{MAPbBr}_{3}$ single crystals with a more appropriate precursor ratio resulted in an almost doubled transparency of the single crystals (which indicates a dramatically reduced defect density in the bulk crystals), whereas the mobility-lifetime products were only slightly improved (by less than $30 \%)^{108}$. In another case, the energy levels of
$\mathrm{MAPbI}_{3}$ were observed to be unchanged even after the loss of about $20 \%$ of the iodine from the films (FIG. $4 \mathrm{~d}$ ) - another indication of defect-tolerant behaviour ${ }^{109}$. Recent studies have also proposed that light illumination could further annihilate the defect pairs in perovskites by prompting ion migration ${ }^{110,111}$, which results in a small effective trap density in optical measurements and in device operation. As a result, the non-radiative recombination losses in perovskite solar cells further decrease under device operation.

The relatively low trap density in polycrystalline OIHPs can also be ascribed to the easy crystallization of perovskite materials. An in situ X-ray scattering study revealed a low crystallization activation energy barrier in the range of $56.6-97.3 \mathrm{~kJ} \mathrm{~mol}^{-1}$, depending on the different lead salts used in the precursors ${ }^{53}$. By contrast, the crystallization activation energy of amorphous silicon (a-Si) reaches $\sim 471 \mathrm{~kJ} \mathrm{~mol}^{-1}$. Benefiting from such a low crystallization energy barrier, perovskite thin films with a

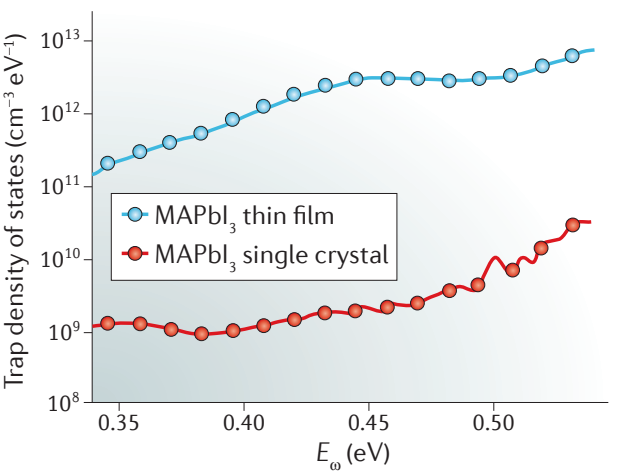

c

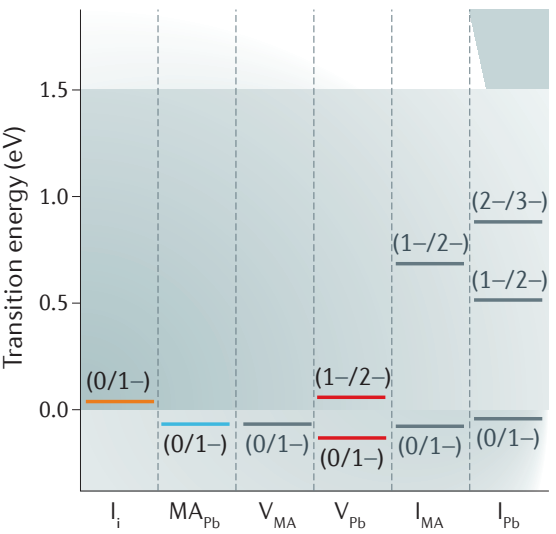

b
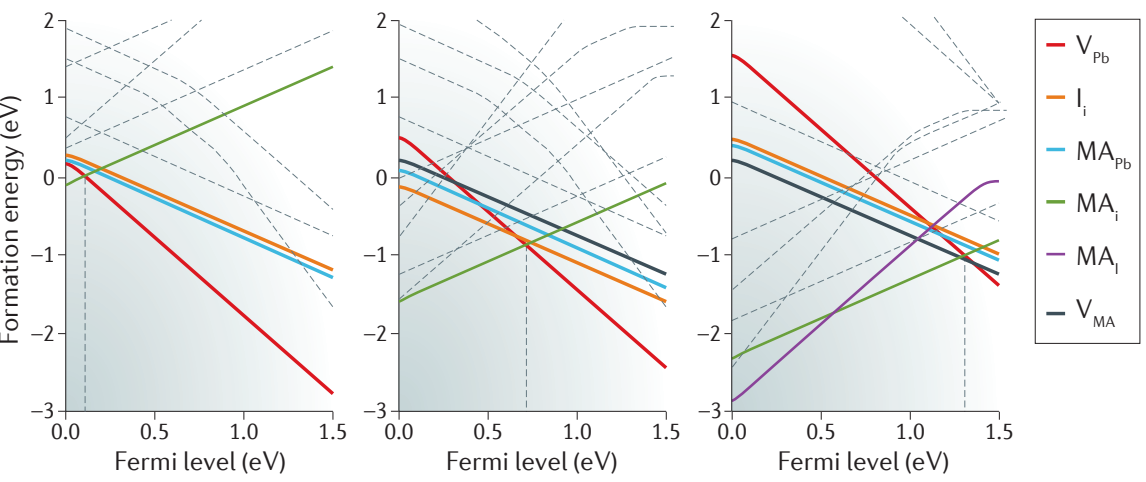

d
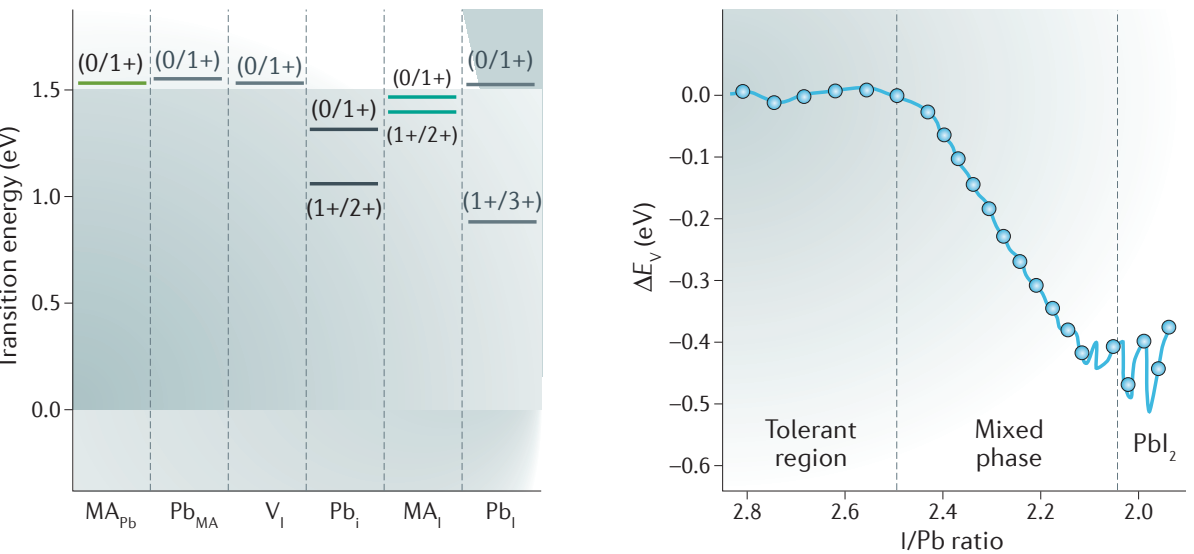

Figure 4 | Defect tolerance of OIHPs. a | Trap density of states in methylammonium lead iodide $\left(\mathrm{MAPbl}_{3}\right)$ polycrystalline thin films and single crystals, where $E_{\omega}$ is the trap depth. $\mathbf{b} \mid$ Formation energies of intrinsic point defects in $\mathrm{MAPbl}_{3}$. Defects with much higher formation energies are displayed as dashed lines, where $\mathrm{MA}_{i}$ is an interstitial MA ion; $I_{\mathrm{i}}$ is an interstitial $I$ ion; $M A_{P b}$ is the $P b$ site occupied by an $M A$ ion; $V_{M A}$ is a vacancy of an $M A$ ion; and $V_{P b}$ is a vacancy of a $\mathrm{Pb}$ ion. c Transition energy levels of intrinsic acceptors (left panel) and intrinsic donors (right panel) in $\mathrm{MAPbl}_{3}$, where $\mathrm{I}_{\mathrm{pb}}$ is the $\mathrm{Pb}$ site occupied by an I ion; $\mathrm{I}_{\mathrm{MA}}$ is the MA site occupied by an I ion; $\mathrm{Pb}_{\mathrm{MA}}$ is the $\mathrm{MA}$ site occupied by a $\mathrm{Pb}$ ion; $\mathrm{V}_{1}$ is a vacancy of an I ion; $\mathrm{Pb}_{i}$ is an interstitial $\mathrm{Pb}$ ion; $\mathrm{MA}_{1}$ is the I site occupied by an $\mathrm{MA}$ ion; and $\mathrm{Pb}_{1}$ is the I site occupied by a $\mathrm{Pb}$ ion. $\mathbf{d}$ | Electronic defect tolerance in $\mathrm{MAPbl}_{3}$. The valence band shift relative to the Fermi level is plotted as a function of the I/Pb ratio. OIHP, organic-inorganic hybrid perovskite. Part a is adapted with permission from REF. 24, AAAS. Parts b and $\mathbf{c}$ are adapted with permission from REF. 55, American Physical Society. Part $\mathbf{d}$ is adapted with permission from REF. 109, American Chemical Society. 
excellent crystallinity and low density of defects could be fabricated by a diverse range of techniques, especially low-temperature solution processes, which would be a competitive edge for commercialization considering the low processing cost.

Surface and grain boundary charge traps. Despite the point-defect tolerance of OIHP materials, the presence of extended defects, such as grain boundaries and interfaces, increases the trap density, as evidenced by the orders-of-magnitude smaller trap density measured in perovskite single crystals. The higher density of defects on grain boundaries and interfaces could be caused by the fact that the surfaces of the material do not necessarily have stoichiometric compositions. For example, evaporation of the organic component from the crystal surface can leave a large density of defects after annealing, while thermal annealing is generally used to increase the grain size of polycrystalline films after film deposition. Even the surfaces of OIHP single crystals can be rich in defects, as demonstrated by narrow-band photodetectors made of single crystals ${ }^{112}$. In this example, surface recombination of photogenerated charges quenched the photoresponse of short-wavelength light, which has a relatively small penetration depth comparable to the thickness of the surface defect layer (FIG. 5a). Only charges generated by light with energy close to the band edge were effectively collected and contributed to the photoresponse, which made the photodetectors sensitive only to light with energy close to the band edge and with a narrow bandwidth of $20 \mathrm{~nm}$. This significant surface recombination in many materials also explains the substantially different photoluminescence lifetimes obtained in perovskite single crystals when the incident light has an apparently different penetration depth (FIC. 5a).

Charge recombination at the surfaces of perovskite materials has proven to be more complicated than initially thought as the study of this process continues. Each facet of the perovskite surfaces is different in terms of defect density. The synthesized $\mathrm{MAPbI}_{3}$ bulk single crystals only have two facets of (100) and (112), whereas the polycrystalline films have many orientations, including (110), (202), (310) and (314), as identified from regular $\mathrm{X}$-ray diffraction patterns. Only the (100) plane is intrinsically non-charged, but other charged planes (or orientations) do exist in polycrystalline film samples. There may be other ions to balance the charges at the surface, either by additional ions from the precursor or ions from the environment. It is also possible that some charged surfaces remain unbalanced. In addition, the binding energy of organic components at each facet can also be different, and thus, the generated defect density should be facet-dependent. This was observed using photoconductive atomic force microscopy, which revealed a $V_{\mathrm{OC}}$ variation of $0.6 \mathrm{~V}$ between different facets on the same grain $^{113}$ (FIG. 5b). However, the maximum $V_{\mathrm{OC}}$ measured at the nanoscale was still smaller than in the macroscopic devices. It is questionable whether the facet-dependent $V_{\text {OC }}$ measured on films without any charge transport layers is correlated to the $V_{\mathrm{OC}}$ of macroscopic devices.
In our recent study, we discovered a similar facet- and grain-dependent $V_{\text {OC }}$ with neat perovskite films deposited on a hole-transport layer and measured with a Kelvin probe force microscope (KPFM); however, covering the perovskite films with an electron-transport layer of PCBM completely wipes out the difference between the grains and facets (J.H. et al. unpublished observations). This agrees well with the very good passivation effect of PCBM in reducing the density of defects of different facets ${ }^{114}$. It is not surprising that the grain orientation is sensitive to the process and composition of the precursors that form the films. There are many cases in which polycrystalline perovskite films have preferred grain orientations (that is, some grains with a particular orientation dominate the polycrystalline films).

In addition to differences between facets, the surface composition of the crystals or thin films may be different from that of the bulk, and thus may influence charge recombination. For example, a very different surface-atom arrangement of as-synthesized $\mathrm{MAPbBr}_{3}$ single crystals was observed compared with that of the free-surface obtained by cleaving ${ }^{115}$. It is not known yet whether the surface composition of the thin films will be dramatically different from the bulk composition, but the loss of volatile organic cations would not be surprising during regular thermal annealing or even storage periods.

The surface recombination rate is also sensitive to the composition and the environmental vapour. Ozone treatment of $\mathrm{MAPbBr}_{3}$ single crystals was shown to significantly enhance the photoluminescence intensity as well as the radiative recombination lifetime from the crystal surface (FIG. 5c), and thus fully recovered the photoresponse in the short wavelength range. This surface passivation by oxidation yielded an extremely low surface recombination rate of $64 \mathrm{~cm} \mathrm{~s}^{-1}$, as derived from photoconductivity measurements. This inspired the later discovery that physical absorption of oxygen and several other gases could also passivate the surface of $\mathrm{MAPbBr}_{3}$, with the demonstration of interesting reversible dimming of the photoluminescence when vacuuming the crystals and brightening when exposed in air. A record low surface recombination rate of $4 \mathrm{~cm} \mathrm{~s}^{-1}$ was derived from the fitting of photoluminescence decay by considering the competition between the surface recombination and drift of the carrier away from the surface. This value is comparable to the best passivated silicon wafers, although the methods of evaluating the surface recombination rate need to be unified in the future for a fair comparison. Similar results were subsequently reported in other studies ${ }^{84,116}$. By contrast, suppression of surface recombination by oxygen was not observed in $\mathrm{MAPbI}_{3}$, and the mechanism of this effect in $\mathrm{MAPbBr}_{3}$ still needs to be elucidated. Nevertheless, the greater tolerance of Br-containing perovskites to oxidation may imply a better stability of such materials in solar cell operations.

The presence of defects inevitably affects the optoelectronic properties of perovskite materials as well as the device performance. The overlooking of surface recombination in early studies of carrier diffusion length resulted in a considerable underestimation of the carrier diffusion length. It is not difficult to distinguish the surface and 


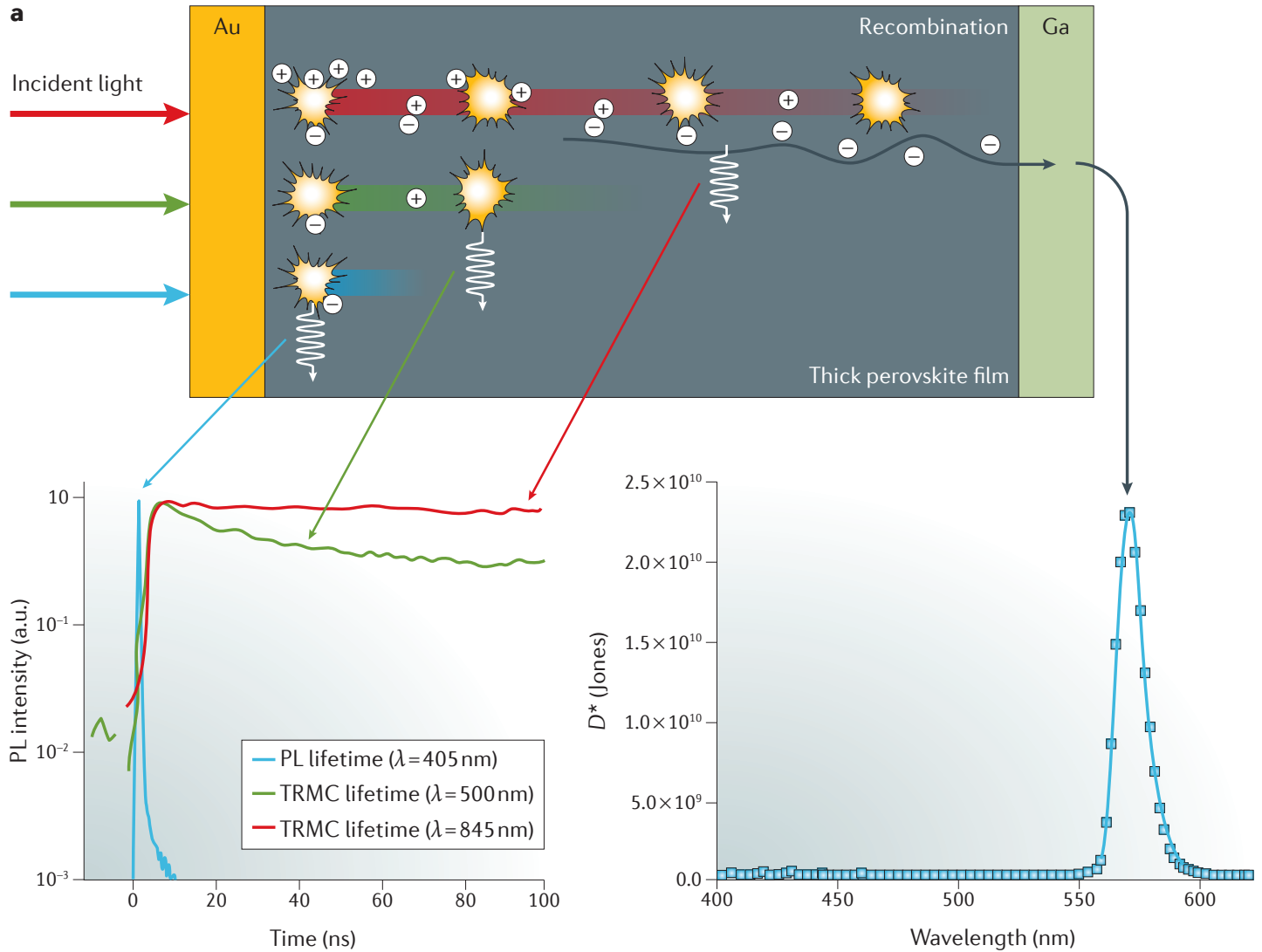

b
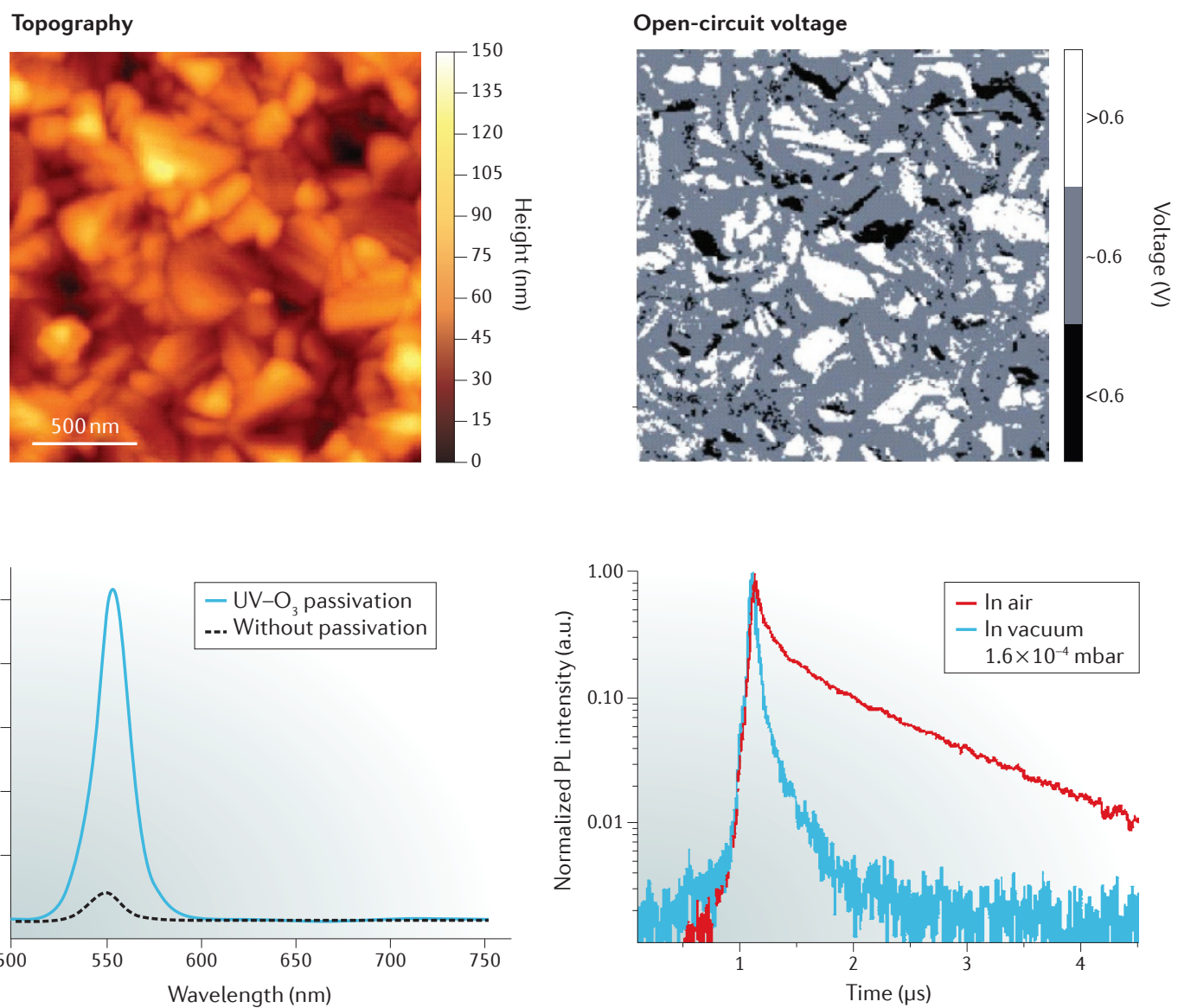
4 Figure 5 | Surface charge recombinations in OIHPs. a | Schematic illustration showing that the charge recombination and collection under above-bandgap excitation is severely affected by surface traps, whereas under below-bandgap excitation it is less susceptible to surface-defect-induced recombination, resulting in significantly different photoluminescence $(\mathrm{PL})$ lifetimes (bottom left part) and narrow-band photodetection (bottom right part). $\mathbf{b} \mid$ Atomic force microscopy image showing the topography of a polycrystalline perovskite thin film. Individual crystal grains and their facets are clearly visible (left part). Corresponding open-circuit voltage $\left(V_{\mathrm{OC}}\right)$ map measured under illumination relative to a forward reference bias of $0.6 \mathrm{~V}$ (right part). Grey areas have $V_{\mathrm{OC}}$ close to the reference bias, white areas above and black areas below. $\mathbf{c |}$ Photoluminescence spectra of a methylammonium lead bromide $\left(\mathrm{MAPbBr}_{3}\right)$ single crystal in vacuum before and after $\mathrm{UV}-\mathrm{O}_{3}$ treatment (left part). Photoluminescence lifetime of the $\mathrm{MAPbBr}_{3}$ single crystal obtained in vacuum and in air (right part). a.u., arbitrary units; OIHP, organic-inorganic hybrid perovskite. Part a (lower left) is adapted with permission from REF. 125, American Chemical Society. Part a (lower right) is adapted with permission from REF. 112, Macmillan Publishers Limited. Part $\mathbf{c}$ is adapted with permission from REF. 108, Macmillan Publishers Limited.

bulk charge recombination in single crystals: by taking into account the diffusion of charges away from the surface, an additional channel of charge density decay the intrinsic carrier diffusion length - can be derived. However, the diffusion of electrons and holes may interact with each other in photoluminescence decay measurements. If the electron mobility is significantly smaller than the hole mobility, the diffusion of holes may be dragged down by trapped electrons to maintain charge neutrality; the measured mobility and diffusion length is thus still limited by charges with lower mobility. To grasp the charge transport and diffusion information of materials, other techniques that are sensitive to the larger mobility-lifetime product, such as terahertz or microwave photoconductivity-based measurements, should be applied to evaluate the majority carrier lifetime and mobility.

Although there is increasing consensus that the surfaces of most OIHP films are defect-rich, whether grain boundaries are benign is still under debate. Theoretical works have shown that 'ideal' grain boundaries — which are formed by connecting two clean surfaces - are electronically benign with negligible deep charge traps. A large $V_{\mathrm{OC}}$ of $1.0-1.1 \mathrm{~V}$ was still achieved in the early stages of perovskite solar cell development when the grains were generally small, which indicates that grain boundaries might not be that defective. By contrast, we also noticed that a higher $V_{\mathrm{OC}}$ was generally achieved with perovskite films that contained additives, such as $\mathrm{Cl}$, which may passivate the grain boundaries. Later studies found that increasing the grain size with various methods generally resulted in large $V_{\mathrm{OC}}$ values $^{117-120}$, although it is not known whether these larger-grain growth techniques also reduce the defect density of the film surface. Direct evidence has been reported for the quenching of charges by grain boundaries by the observation of dimmer photoluminescence, and quicker photoluminescence decay at grain boundaries with grain-scale photoluminescence imaging and local photoluminescence decay measurements ${ }^{121}$. However, the possible band bending close to the grain boundaries adds complexity to the interpretation of photoluminescence intensity and lifetime variation, and thus whether grain boundaries are benign remains an open question.
It should be noted that grain boundaries in real OIHP films are highly likely to be non-ideal. Similar to surfaces, excess intrinsic and extrinsic impurities may be segregated at grain boundaries, affecting the electronic properties of the thin films.

Trap density characterization. Accurate characterization of charge trap density in OIHP materials is a crucial step towards understanding the associated optoelectronic properties of these materials, but is still highly challenging because of the unknown charge defect configurations. The earliest direct measurement of charge trap density was carried out with thermal admittance spectroscopy, which gives the densities of charge traps at different energy depths. The presence of a large density of charge traps was shown to exist in as-grown perovskite films, which reduced device efficiency and caused photocurrent hysteresis ${ }^{102}$. The physical attachment of $\mathrm{MAPbI}_{3}$ polycrystalline films with PCBM and $\mathrm{C}_{60}$ was found to reduce both shallower and deeper traps by about one to two orders of magnitude, indicating that the majority of the charge traps are located at the surface of the perovskite films ${ }^{102}$ (FIG. 6a). In an initial optical study, a bulk trap density of $\sim 5 \times 10^{16} \mathrm{~cm}^{-3}$ was estimated from radiative recombination decay by assuming trap state recombination is much slower than band-edge radiative recombination; the surface trap density was found to be about threefold higher ${ }^{122}$. The short radiative recombination lifetimes measured in these studies indicate a relatively low quality of polycrystalline films. To understand the intrinsic charge trap density, perovskite single crystals have been studied, whereby a trap-filled space-charge-limited current (FIG. 6b) was used to estimate the trap density ${ }^{24,61,123,124}$. The yielded charge trap densities in $\mathrm{MAPbI}_{3}$ and $\mathrm{MAPbBr}_{3}$ single crystals were surprisingly small, of the order of $10^{9}-10^{10} \mathrm{~cm}^{-3}$, which is comparable to those of the best intrinsic silicon and GaAs. Thermal admittance spectroscopy showed a similar result of a lower trap density in single crystals by two to three orders of magnitude compared with polycrystalline films. A trap density of $1.5 \times 10^{13} \mathrm{~cm}^{-3}$ was directly measured in the $\mathrm{MAPbI}_{3}$ single crystals by time resolved microwave conductivity ${ }^{125}$. Because all crystals used in this study were the same, this excluded any effects caused by differences in crystal quality. Thus, different measurement methods examine different processes or different types of traps. It is highly likely that some charge traps did not have a significant role in determining the dark current, although they strongly impact the photocurrent signal.

Knowing the trap density of electrons and holes is important for understanding the limitations of these materials, which can guide the design of new perovskite materials with improved properties. However, most current measurements only reveal the total trap density. To separate the electron- and hole-conduction properties, the simple photoconductor structure shown in FIG. $6 \mathrm{c}$ was combined with charge acceptor layers that remove one type of charge and leave the other type in the perovskite layer ${ }^{126}$. Because charge transport materials have orders-of-magnitude smaller mobility 


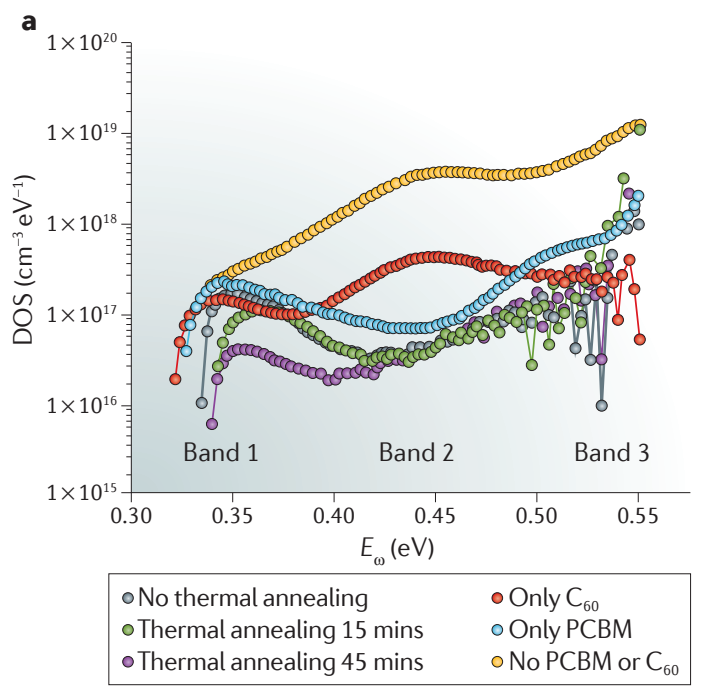

C

\begin{tabular}{|c|c|}
\hline \multicolumn{1}{|c|}{ PMMA, Spiro or PCBM } & $\mathrm{Au}$ \\
\hline $\mathrm{MAPbl}_{3}$ \\
\hline Substrate \\
\hline
\end{tabular}

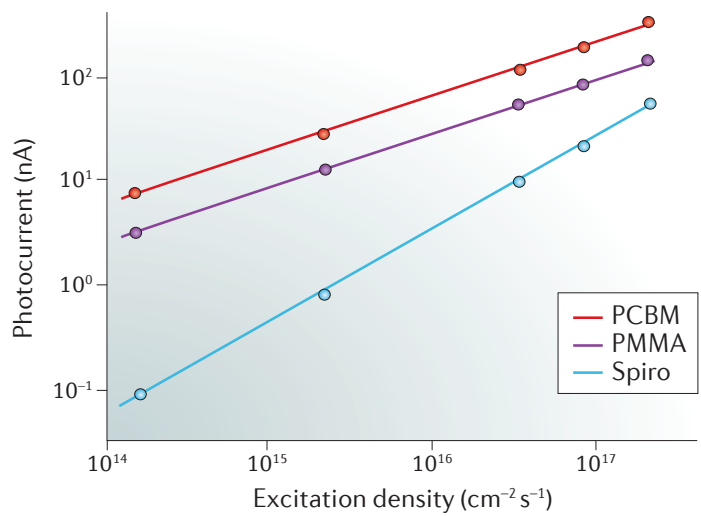

b

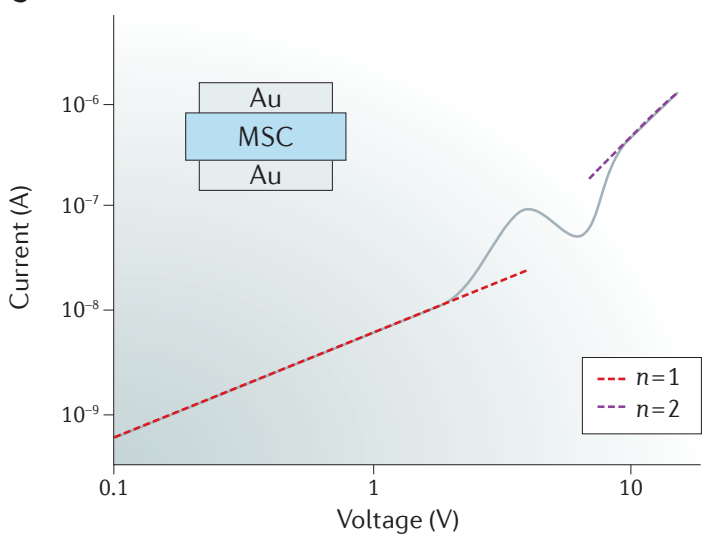

d
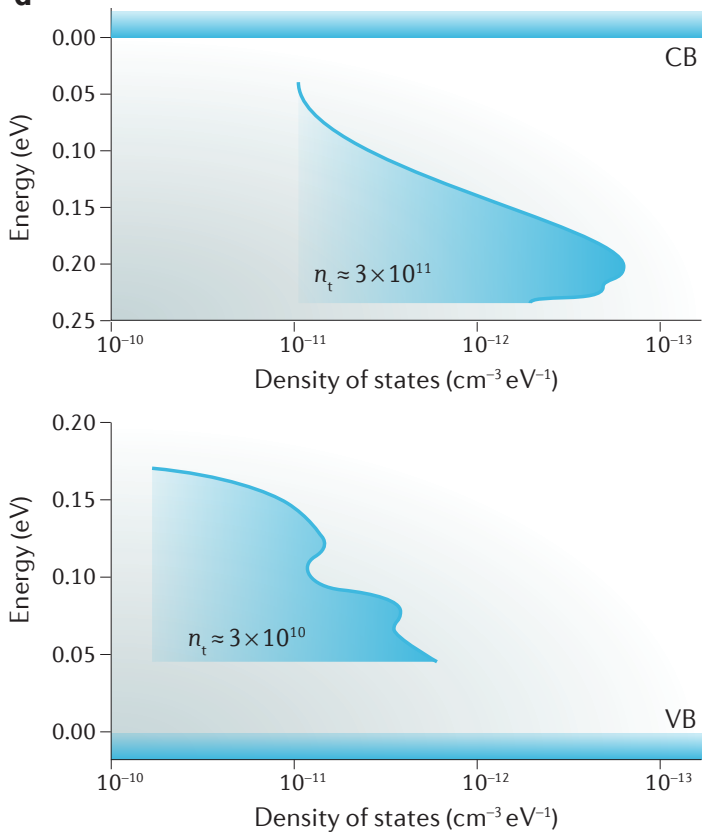

Figure 6 | Charge trap densities and surface passivation of OIHPs. a | Reduced density of shallow and deep traps (where $E_{\omega}$ is trap depth) by $\mathrm{C}_{60}$ and phenyl- $\mathrm{C}_{61}$-butyric acid methyl ester (PCBM) surface passivation, where band 1 includes shallow trap states with depths of $0.35-0.40 \mathrm{eV}$, band 2 includes trap states with depths of $0.40-0.50 \mathrm{eV}$, and band 3 includes deep trap states with depths over $0.50 \mathrm{eV}$. $\mathbf{b} \mid$ Current-voltage curve for a methylammonium lead iodide single crystal (MSC) device. The inset shows the device structure. Three regions can be identified according to different values of the exponent, $n: n=1$ is the ohmic region; $n=2$ is the space charge limited current (SCLC) region, and in between is the trap-filled limited region. $\mathbf{c}$ /Schematic of the symmetric laterally contacted device (top part). Photocurrent $(10 \mathrm{~V})$ as a function of intensity of above-bandgap $(690 \mathrm{~nm})$ excitation for devices shown in part $\mathbf{b}$ covered by a hole-accepting Spiro-OMeTAD layer, an electron-accepting PCBM layer or an inert poly(methyl methacrylate) (PMMA) layer. $\mathbf{d}$ |Density of the trap states $\left(n_{t}\right)$ within the bandgap: trap states are identified close to the conduction band (CB) and the valence band (VB). OIHP, organic-inorganic hybrid perovskite. Part $\mathbf{a}$ is adapted with permission from REF. 114, Macmillan Publishers Limited. Part b is adapted with permission from REF. 24, AAAS. Part $\mathbf{c}$ is adapted with permission from REF. 126, Royal Society of Chemistry. Part d is adapted with permission from REF. 135, Wiley-VCH.

than polycrystalline perovskite thin films, the photoconductivity is dominated by the contribution from the perovskite layer. One prerequisite for this measurement is that the lifetime of charge-separated states is sufficiently long so that a sizeable photoconductivity can be measured with a laterally structured photoconductor, because the photoconductivity is determined as the product of the carrier mobility and the carrier recombination lifetime $e^{127-129}$. Charge-separated states last for several hundred microseconds at the interfaces of $\mathrm{MAPbI}_{3}$ and the electron acceptors $\mathrm{PCBM}^{130}$ and $\mathrm{TiO}_{2}$ (REF. 131), and single-walled carbon nanotube hole acceptors ${ }^{131,132}$. Steady-state photoconductivity measurements revealed a much higher hole conductivity than electron conductivity (FIG. 6c), which was attributed to a weaker trapping effect to holes in $\mathrm{MAPbI}_{3}$ than to electrons ${ }^{126}$. This is consistent 
with the measured larger hole mobility in single crystals of $\mathrm{MAPbI}_{3}$ and $\mathrm{MAPbBr}_{3}$ (REF. 24), and also agrees with the slight $\mathrm{p}$-doping of these two materials in single crystals and polycrystalline films $\mathrm{s}^{24,108,133}$. Nevertheless, the difference between the electron and hole mobilities and/ or lifetimes may be exaggerated in these studies, because the gold electrodes used may not effectively inject electrons to provide photoconductive gain, and the deposition of gold on the perovskite may also generate electron traps in the perovskite layer underneath the electrode. Thus, a better choice of electrodes in electron- and holeonly devices gives more accurate electron- and hole-only currents $^{134}$. In this particular study, although the hole current was several orders of magnitude larger than the electron current at high device bias $(1-1.5 \mathrm{~V})$, the quality of the perovskite films was not uniform, because the substrates (for example, surface energy) strongly affected the grain size and crystallinity of the perovskite films ${ }^{120}$. In another example, electron- and hole-only devices were constructed to measure the electron and hole trap distributions in single-crystal $\mathrm{MAPbI}_{3}$ (REF. 135). As shown in FIG. $6 \mathrm{~d}$, a low trap density of $3 \times 10^{10} \mathrm{~cm}^{-3}$ was derived for holes, whereas the electron trap density was over tenfold larger. The application of a hole-injection layer of $\mathrm{MoO}_{3}$ may affect the accuracy of this measurement for hole-only devices, because $\mathrm{MoO}_{3}$ undergoes destructive chemical reactions with the perovskite ${ }^{136}$.

The charge trap density was directly measured at the surface of perovskite films with an all-optical method ${ }^{137}$. The enhanced absorption cross-section of the trap states by approximately three orders of magnitude allowed the direct observation of a high density of deep trap states by UPS using a low-energy ultraviolet source. A large density of hole traps was observed, which extended to the Fermi level for $\mathrm{MAPbI}_{3}$ films formed by thermal evaporation. The authors speculated that a similarly high density of electron traps should exist below the valence band. It is frequently reported that $\mathrm{MAPbI}_{3}(\mathrm{Cl})$ films made of precursors with $\mathrm{Cl}$ have longer photoluminescence radiative recombination lifetimes and better thermal stability than $\mathrm{MAPbI}_{3}$ films, although the percentage of $\mathrm{Cl}$ that remains in the films is still unclear ${ }^{88,138,139}$. The fact that a comparable trap density was observed for $\mathrm{MAPbI}_{3}$ and $\mathrm{MAPbI}_{3}(\mathrm{Cl})$ should remind researchers to pay particular attention when using vacuum-based UPS measurements for surface analysis of most perovskite polycrystalline films because of the low surface stability of perovskites in vacuum. We have observed the decomposition of uncovered $\mathrm{MAPbI}_{3}$ surfaces in low vacuum over the course of several hours at the macroscopic level, which was greatly accelerated by illumination and even long UPS radiation (for example, see the supplementary information of REF. 96).

The role of ion migration. Ion migration is a unique property of hybrid perovskites and is not present in other photovoltaic materials. Although ion migration has been reported to affect device operation in various ways, we instead focus on the beneficial role of ion migration on device efficiency and stability. Detailed reviews on the mechanism and effects of ion migration can be found elsewhere ${ }^{140-142}$. We have proposed and established that ion migration and associated ion accumulation could cause a chemical doping effect, which is a natural choice for the formation of an ohmic contact for high-efficiency solar cells with a p-i-n or n-i-p structure ${ }^{71}$ (FIG. 7a). Recently, an anomalous photovoltaic effect in laterally structured perovskite solar cells was discovered, whereby the largest obtained $V_{\mathrm{OC}}$ of $18 \mathrm{~V}$ in a device with an electrode spacing of $100 \mu \mathrm{m}$ significantly exceeded the ratio of energy bandgaps $\left(E_{\mathrm{g}}\right)$ of the perovskite materials to electron charge ${ }^{96}$ (that is, $V_{\mathrm{OC}}>E_{\mathrm{g}} / q$, FIG. $7 \mathrm{~b}$ ). This effect was observed with a group of perovskite materials as the photoactive layers, including $\mathrm{MAPbI}_{3}, \mathrm{MAPbBr}_{3}$ and $\mathrm{CsPbBr}$. Switchable diodes and an anomalous photovoltaic effect have been observed in ferroelectric materials ${ }^{143}$; however, the anomalous photovoltaic effect in these hybrid perovskite devices was shown to be caused by the ion migration effect ${ }^{71,96,141,144}$. A KPFM study showed that the accumulation of ions and the associated doping effect is sufficient to cause large band bending around the area of accumulation, and also to form tunnelling junctions that may connect multiple cells ${ }^{96}$. Randomly dispersed tunnelling junctions in polycrystalline perovskites enable the accumulation of photovoltage on a microscale, leading to a $V_{\mathrm{OC}}$ output that is proportional to the electrode spacing. This finding further confirms the potential of ion manipulation in tuning the electrical properties of perovskite films (or interfaces) to enhance the device performance, despite the fact that the current random-dispersed tunnelling junction is structurally different from the continuous tunnelling junctions in conventional tandem devices.

There is no reason that the accumulation of ions would not occur in regular, operating solar cells, because ion migration can be significantly enhanced under illumination. The accumulation of ions under working conditions was shown to actually enhance the device efficiency by providing an additional electric field to facilitate charge extraction ${ }^{145}$. This may contribute to enhanced device efficiency over time. Nevertheless, the ion back-diffusion that occurs when the devices are kept in the dark would negate the light-induced diffusion. Recently, a considerable PCE enhancement by ion accumulation was reported by poling and measuring devices at lower temperature to effectively freeze ion back-diffusion (from 14\% at room temperature to $19 \%$ at $170 \mathrm{~K}, \mathrm{FIG} .7 \mathrm{a})^{146}$. The critical temperature for a stabilized poling state was close to room temperature $(250 \mathrm{~K})^{146}$. This implies that ion migration could be beneficial for room temperature device operation if the ion migration activation energy $\left(E_{\mathrm{a}}\right)$ could be doubled such that the ion migration rate can be reduced by several orders of magnitude, if considering that the ion migration rate decreases exponentially with the ratio of $E_{\mathrm{a}} / k T$, where $k T$ is the thermal energy ${ }^{141,142}$.

There is little doubt that ion migration can impair the long-term device stability of perovskite solar cells under continuous operation by damaging the perovskite layer or other layers. Nevertheless, some interesting studies have shown that the short-term stability actually benefits from ion migration in the perovskite layer of devices operating under cycled illumination. A common observation for devices that are degraded after 

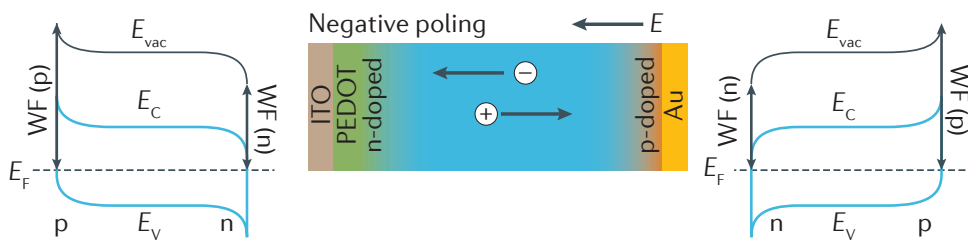

b Anomalous photovoltaic effect

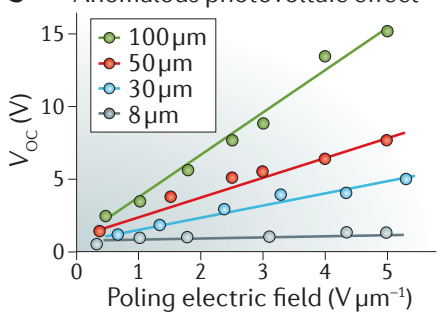

Dispersed tunnelling junction

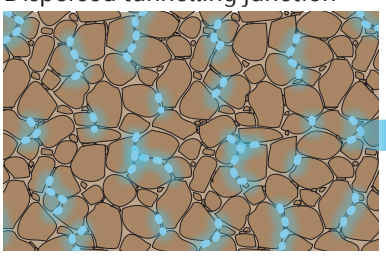

$\mathrm{MAPbBr}_{3}$ grains

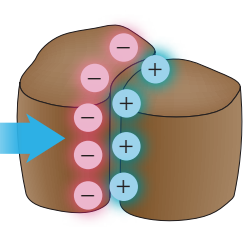

Grain boundaries with accumulated ions

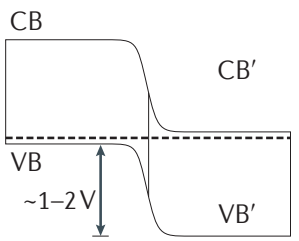

$\mathrm{VB}^{\prime}$ c Typical situation 1
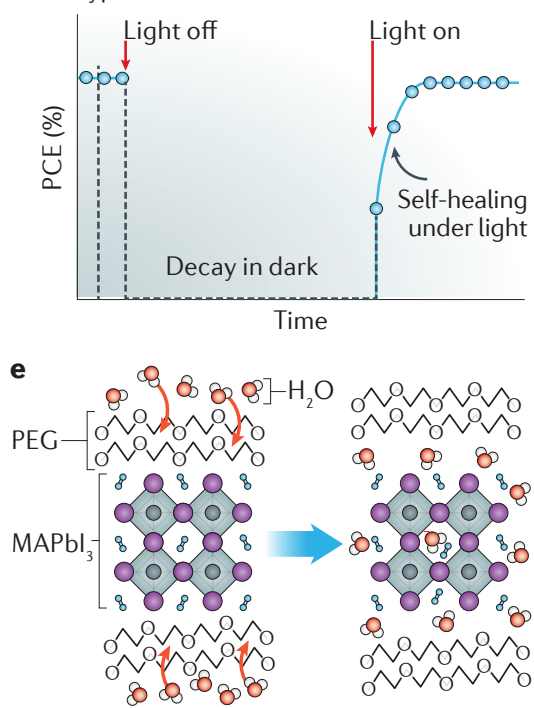

Typical situation 2
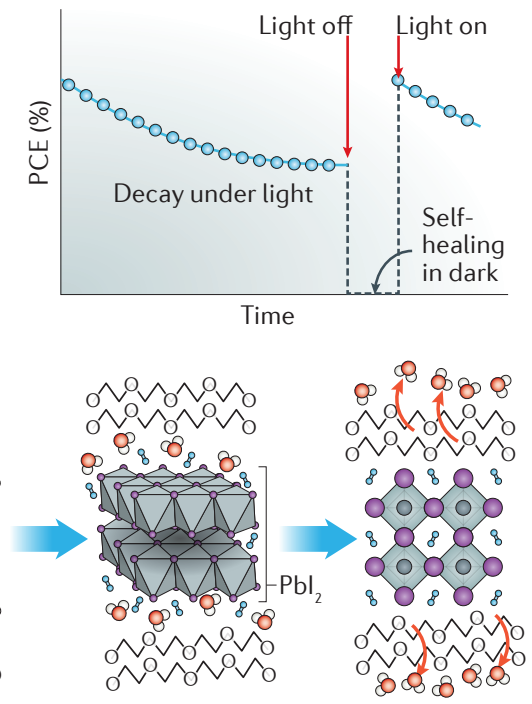

d $t<0 ; n_{\mathrm{t}}>10^{17} \mathrm{~cm}^{-3}$

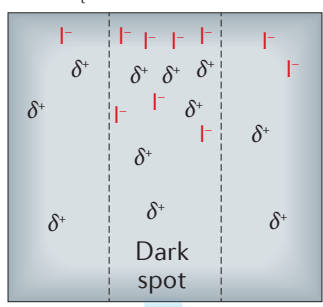

Illumination

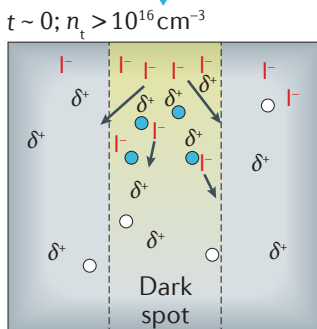

Figure 7 | Ion migration in OIHPs. a | Schematic illustration of a methylammonium lead iodide $\left(\mathrm{MAPbl}_{3}\right)$ solar cell with redistributed ions which form $p$ - and n-type doping regions in situ and provide ohmic contact interfaces, where $E_{c}$ is the conduction band energy, $E_{\mathrm{F}}$ is the Fermi energy, $E_{\mathrm{v}}$ is the valence band energy and $E_{\mathrm{vac}}$ is the vacuum energy. $\mathbf{b} \mid$ Schematic illustration of an electrically poled polycrystalline perovskite film with a dispersed tunnelling junction formed by the accumulation of local ions, which causes dramatic band bending at grain boundaries and hence leads to an anomalous photovoltaic effect $\left(V_{\text {OC }}\right.$ is the open-circuit voltage). $c \mid$ Typical power conversion efficiency (PCE)-time plots of perovskite solar cells with different type of self-healing behaviour. In typical situation 1, the device shows a PCE drop after being stored in the dark, which is followed by gradual PCE self-healing after the light was turned on; in typical situation 2, the device shows a gradual PCE drop in continuous operation and then a partially or completely self-healing process once it was stored in dark. $\mathbf{d} \mid$ Schematic illustration showing the reduced trap concentration in $\mathrm{MAPbl}_{3}$ films due to the light-induced redistribution of mobile ions: that is, the iodide vacancies $\left(\delta^{+}\right)$are filled by excessive iodide ions $\left(I^{-}\right)$. e | Schematic illustration of the mechanisms of self-healing in $\mathrm{MAPbl}_{3}$ solar cells with polyethylene glycol (PEG) used to fill in the grain boundaries. The PEG traps the escaped $\mathrm{MA}^{+}$ions in high-humidity environments, and the trapped $\mathrm{MA}^{+}$ released from PEG converts $\mathrm{Pbl}_{2}$ grains to $\mathrm{MAPbl}_{3}$ grains when the humidity decreases. $\mathrm{CB}$, conductance band; $\mathrm{VB}$, valence band; ITO, indium tin oxide; $n_{\mathrm{t}}$, trap density; OIHP, organic-inorganic hybrid perovskite; PEDOT, poly(3,4-ethylenedioxythiophene); WF, work function. Part a is adapted with permission from REF. 71, Macmillan Publishers Limited. Part $\mathbf{b}$ is adapted with permission from REF. 96, AAAS. Part $\mathbf{d}$ is adapted with permission from REF. 111, Macmillan Publishers Limited. Part e is adapted with permission from REF. 151, Macmillan Publishers Limited.

light exposure is that they can partially or completely recover their efficiency after storage in the dark (FIG. 7c). Although there are differences between studies in terms of the conditions for device self-healing (that is, in the dark $^{147}$ or under light-soaking ${ }^{148}$ ), it is possible that the self-healing effect is related to the role of ion diffusion in the destruction and reconstruction of perovskite crystals (FIG. 7d). Mobile ions could have a role in the curing of local lattice distortion or decomposition because the timescale for ions travelling through the film (seconds or minutes) is much shorter than that of film degradation (days or months) ${ }^{71,149}$, and because the conversion 
between the $\mathrm{MAPbI}_{3}$ and $\mathrm{PbI}_{2}$ phases enabled by ion migration is fairly reversible ${ }^{150}$. An important issue for self-healing caused by ion migration is that the mobile ions should be sealed to prevent volatilization or chemical reaction. Methods such as filling the grain boundaries in $\mathrm{MAPbI}_{3}$ films with polyethylene glycol have been reported to prevent the loss of volatile MA cations and thus take advantage of the self-healing effect to extend device stability ${ }^{151}$ (FIG. 7e).

\section{Conclusion and outlook}

There has been tremendous progress in the past few years in understanding the unique properties of hybrid perovskites and why these materials can make very efficient solar cells. In this Review, we have provided a critical discussion about the intrinsic electro- and optoelectronic properties of OIHP materials and tried to correlate these properties with the device working mechanisms and device performance. There is already consensus that many key material properties of solution-processed ionic OIHP films, such as light absorption, charge trap depth, exciton binding energies and charge diffusion length are comparable with or superior to the best properties of meticulously fabricated, covalently bonded photovoltaic materials (for example, $\mathrm{Si}, \mathrm{GaAs}$ and CdTe) by hightemperature and vacuum methods. Many unique properties, such as a defect-tolerant nature, charge screening and ion migration, are now also well established.

Despite this progress, many important questions remain unanswered. Among all the interesting properties, the ferroelectric nature of $\mathrm{MAPbI}_{3}$ needs to be clarified. In this regard, studying the effects of tuning by the partial substitution of cations with $\mathrm{FA}^{+}, \mathrm{Rb}^{+}, \mathrm{Cs}^{+}$or $\mathrm{Co}^{2+}$ will be interesting, and itself may provide a pathway to determine the function of the ferroelectric dipoles. Recent progress in further boosting the device efficiency heavily relies on composition engineering, whereby additional ions are introduced. The function of the added ions needs to be thoroughly understood to recognize the 'limitations' of the simple perovskite material of $\mathrm{MAPbI}_{3}$. Another important direction will be to determine a possible inherent correlation between the observed unique properties, including electric, mechanical, optical and spin properties. Although the large lattice constant and soft nature of OIHP materials gives rise to a pronounced ion migration effect, the ways in which these properties affect the favourably large material dielectric constant and structural stability are not clear yet. The mechanism of individual ion migration still needs to be thoroughly characterized. In addition, we need to gain an understanding of how individual and collaborative ion migration affects carrier recombination in the photoactive layer and at interfaces, as well as a better understanding of charge collection, current-voltage hysteresis and perovskite stability. It is almost without any doubt that exciting and new properties will continue to be discovered in OIHP materials. Our advanced understanding of the unique optoelectronic properties of OIHP materials may now lead to the discovery of promising non-toxic and air-stable perovskite light absorbers.
1. Li, X. et al. A vacuum flash-assisted solution process for high-efficiency large-area perovskite solar cells. Science 353, 58-62 (2016)

2. Baikie, T. et al. Synthesis and crystal chemistry of the hybrid perovskite $\left(\mathrm{CH}_{3} \mathrm{NH}_{3}\right) \mathrm{Pbl}_{3}$ for solid-state sensitised solar cell applications. J. Mater. Chem. A 1 5628-5641 (2013).

A pioneering study of the thermal stability and crystallography of perovskite materials, especially $\mathrm{MAPbl}_{3}$.

3. Divitini, G. et al. In situ observation of heat-induced degradation of perovskite solar cells. Nat. Energy 1, 15012 (2016)

4. Stoumpos, C. C., Malliakas, C. D. \& Kanatzidis, M. G. Semiconducting tin and lead iodide perovskites with organic cations: phase transitions, high mobilities, and near-infrared photoluminescent properties. Inorg. Chem. 52, 9019-9038 (2013).

A pioneering study of all kinds of optoelectronic properties of $\mathrm{Pb}$-based and $\mathrm{Sn}$-based hybrid (and all-inorganic) perovskite materials.

5. Niu, G., Guo, X. \& Wang, L. Review of recent progress in chemical stability of perovskite solar cells. J. Mater. Chem. A 3, 8970-8980 (2015).

6. Jensen, N., Hausner, R., Bergmann, R., Werner, J. \& Rau, U. Optimization and characterization of amorphous/crystalline silicon heterojunction sola cells. Prog. Photovoltaics 10, 1-14 (2002).

7. Xiao, Z. et al. Solvent annealing of perovskite-induced crystal growth for photovoltaic-device efficiency enhancement. Adv. Mater. 26, 6503-6509 (2014).

8. Lee, M. M., Teuscher, J., Miyasaka, T., Murakami, T. N. $\&$ Snaith, H. J. Efficient hybrid solar cells based on meso-superstructured organometal halide perovskites. Science 338, 643-647 (2012).

9. Zhao, J., Wang, A., Green, M. A. \& Ferrazza, F. 19.8\% efficient "honeycomb" textured multicrystalline and $24.4 \%$ monocrystalline silicon solar cells. Appl. Phys. Lett. 73, 1991-1993 (1998).

10. Brivio, F., Butler, K. T., Walsh, A. \& Van Schilfgaarde, M. Relativistic quasiparticle selfconsistent electronic structure of hybrid halide perovskite photovoltaic absorbers. Phys. Rev. B 89 155204 (2014)

11. Green, M. A., Ho-Baillie, A. \& Snaith, H. J. The emergence of perovskite solar cells. Nat. Photonics $\mathbf{8}$, 506-514 (2014).

12. Yin, W. J., Shi, T. \& Yan, Y. Unique properties of halide perovskites as possible origins of the superior solar cell performance. Adv. Mater. 26, 4653-4658 (2014).

13. Giebink, N. C., Wiederrecht, G. P., Wasielewski, M. R. \& Forrest, S. R. Thermodynamic efficiency limit of excitonic solar cells. Phys. Rev. B 83, 195326 (2011).

14. Shaklee, K. \& Nahory, R. Valley-orbit splitting of free excitons? The absorption edge Si. Phys. Rev. Lett. 24, 942 (1970)

15. Fehrenbach, G., Schafer, W. \& Ulbrich, R. Excitonic versus plasma screening in highly excited gallium arsenide. J. Lumin. 30, 154 (2012).

16. Lin, Q., Armin, A., Nagiri, R C. R., Burn, P. L. $\delta$ Meredith, P. Electro-optics of perovskite solar cells. Nat. Photonics 9, 106-112 (2015).

17. D'Innocenzo, V. et al. Excitons versus free charges in organo-lead tri-halide perovskites. Nat. Commun. 5 3586 (2014)

18. Hirasawa, M., Ishihara, T., Goto, T., Uchida, K. \& Miura, N. Magnetoabsorption of the lowest exciton in perovskite-type compound $\left(\mathrm{CH}_{3} \mathrm{NH}_{3}\right) \mathrm{Pbl}_{3}$. Phys. $B$ : Condens. Matter 201, 427-430 (1994).

19. Tanaka, K. et al. Comparative study on the excitons in lead-halide-based perovskite-type crystals $\mathrm{CH}_{3} \mathrm{NH}_{3} \mathrm{PbBr}_{3} \mathrm{CH}_{3} \mathrm{NH}_{3} \mathrm{Pbl}_{3}$. Solid State Commun. 127 619-623 (2003).

The $E_{\mathrm{B}}$ of $\mathrm{MAPbl}_{3}$ and $\mathrm{MAPbBr}_{3}$ were accurately identified in this magnetoabsorption study.

20. Ishihara, T. Optical properties of Pbl-based perovskite structures. J. Lumin. 60, 269-274 (1994)

21. Saba, M. et al. Correlated electron-hole plasma in organometal perovskites. Nat. Commun. 55049 (2014).

22. Hu, M. et al. Distinct exciton dissociation behavior of organolead trihalide perovskite and excitonic semiconductors studied in the same system. Small 11 2164-2169 (2015).

23. Grancini, G. et al. Role of microstructure in the electron-hole interaction of hybrid lead halide perovskites. Nat. Photonics 9, 695-701 (2015).

24. Dong, Q et al. Electron-hole diffusion lengths $>175 \mu \mathrm{m}$ in solution-grown $\mathrm{CH}_{3} \mathrm{NH}_{3} \mathrm{Pbl}_{3}$ single crystals. Science 347, 967-970 (2015). This study disclosed the intrinsic property of perovskites that enabled the development of solar cell radiation detectors.

25. Nah, S. et al. Spatially segregated free-carrier and exciton populations in individual lead halide perovskite grains. Nat. Photonics 11, 285-288 (2017).

26. Yang, Y. et al. Observation of a hot-phonon bottleneck in lead-iodide perovskites. Nat. Photonics 10, 53-59 (2016).

The first report of long hot carriers in perovskites that may result in real applications.

27. Yang Y et al. Large polarization-dependent exciton optical Stark effect in lead iodide perovskites. Nat. Commun. 712613 (2016)

28. Dou, L. et al. Atomically thin two-dimensional organicinorganic hybrid perovskites. Science 349 1518-1521 (2015).

29. Stoumpos, C. C. et al. Ruddlesden-Popper hybrid lead iodide perovskite 2D homologous semiconductors. Chem. Mater. 28, 2852-2867 (2016).

30. Giovanni, D. et al. Tunable room-temperature spinselective optical Stark effect in solution-processed layered halide perovskites. Sci. Adv. 2, e1600477 (2016).

31. Milot, R. L. et al. Charge-carrier dynamics in $2 \mathrm{D}$ hybrid metal-halide perovskites. Nano Lett. 16, 7001-7007 (2016)

32. Wu, X., Trinh, M. T. \& Zhu, X.-Y. Excitonic many-body interactions in two-dimensional lead iodide perovskite quantum wells. J. Phys. Chem. C 119, 14714-14721 (2015).

33. Blancon, J.-C. et al. Extremely efficient internal exciton dissociation through edge states in layered 2D perovskites. Science 355, 1288-1292 (2017). 
34. Yaffe, O. et al. Excitons in ultrathin organic-inorganic perovskite crystals. Phys. Rev. B 92,045414 (2015)

35. Tanaka, K. et al. Image charge effect on twodimensional excitons in an inorganic-organic quantum-well crystal. Phys. Rev. B 71, 045312 (2005).

36. Johnston, M. B. \& Herz, L. M. Hybrid perovskites for photovoltaics: charge-carrier recombination, diffusion, and radiative efficiencies. Acc. Chem. Res. 49, 146-154 (2015)

37. Milot, R. L., Eperon, G. E., Snaith, H. J., Johnston, M. B. \& Herz, L. M. Temperature-dependent charge-carrier dynamics in $\mathrm{CH}_{3} \mathrm{NH}_{3} \mathrm{Pbl}_{3}$ perovskite thin films. Adv. Funct. Mater. 25, 6218-6227 (2015).

38. Wehrenfennig, C., Eperon, G. E., Johnston, M. B. Snaith, H. J. \& Herz, L. M. High charge carrier mobilities and lifetimes in organolead trihalide perovskites. Adv. Mater. 26, 1584-1589 (2014)

39. Wehrenfennig, C., Liu, M., Snaith, H. J.,

Johnston, M. B. \& Herz, L. M. Charge-carrier dynamics in vapour-deposited films of the organolead halide perovskite $\mathrm{CH}_{3} \mathrm{NH}_{3} \mathrm{Pbl}_{3-x} \mathrm{Cl}_{x}$. Energy Environ. Sci. 7 , 2269-2275 (2014).

40. Yamada, Y., Nakamura, T., Endo, M., Wakamiya, A. \& Kanemitsu, $Y$. Photocarrier recombination dynamics in perovskite $\mathrm{CH}_{3} \mathrm{NH}_{3} \mathrm{Pbl}_{3}$ for solar cell applications. J. Am. Chem. Soc. 136, 11610-11613 (2014).

41. Stranks, S. D. et al. Recombination kinetics in organic-inorganic perovskites: excitons, free charge, and subgap states. Phys. Rev. Appl. 2, 034007 (2014).

42. Manser, J. S. \& Kamat, P. V. Band filling with free charge carriers in organometal halide perovskites. Nat. Photonics 8, 737-743 (2014).

43. Deschler, F. et al. High photoluminescence efficiency and optically pumped lasing in solution-processed mixed halide perovskite semiconductors. J. Phys. Chem. Lett. 5, 1421-1426 (2014)

44. Savenije, T. J. et al. Thermally activated exciton dissociation and recombination control the carrier dynamics in organometal halide perovskite. J. Phys. Chem. Lett. 5, 2189-2194 (2014)

45. Oga, H., Saeki, A., Ogomi, Y., Hayase, S. \& Seki, S. Improved understanding of the electronic and energetic landscapes of perovskite solar cells: high local charge carrier mobility, reduced recombination, and extremely shallow traps. J. Am. Chem. Soc. 136, 13818-13825 (2014).

46. Chen, Y. et al. Extended carrier lifetimes and diffusion in hybrid perovskites revealed by Hall effect and photoconductivity measurements. Nat. Commun. 7 12253 (2016).

47. Wang, Q., Dong, Q., Li, T., Gruverman, A. \& Huang, J. Thin insulating tunneling contacts for efficient and water-resistant perovskite solar cells. Adv. Mater. $\mathbf{2 8}$ 6734-6739 (2016).

48. Pérez-del-Rey, D. et al. Strontium insertion in methylammonium lead iodide: long charge carrier lifetime and high fill-factor solar cells. Adv. Mater. $\mathbf{2 8}$, 9839-9845 (2016)

49. deQuilettes, D. W. et al. Photoluminescence lifetimes exceeding $8 \mu$ s and quantum yields exceeding $30 \%$ in hybrid perovskite thin films by ligand passivation. ACS Energy Lett. 1, 438-444 (2016).

50. Blackburn, J. L. Semiconducting single-walled carbon nanotubes in solar energy harvesting. ACS Energy Lett. 2, 1598-1613 (2017)

51. Zheng, F., Tan, L. Z., Liu, S. \& Rappe, A. M. Rashba spin-orbit coupling enhanced carrier lifetime in $\mathrm{CH}_{3} \mathrm{NH}_{3} \mathrm{Pbl}_{3}$. Nano Lett. 15, 7794-7800 (2015)

52. Nelson, R. $\&$ Sobers, R. Minority-carrier lifetimes and internal quantum efficiency of surface-free GaAs. J. Appl. Phys. 49, 6103-6108 (1978).

53. Moore, D. T. et al. Crystallization kinetics of organicinorganic trihalide perovskites and the role of the lead anion in crystal growth. J. Am. Chem. Soc. 137 2350-2358 (2015).

54. Köster, U. Crystallization of amorphous silicon films. Phys. Status Solidi A 48, 313-321 (1978).

55. Yin, W.-J., Shi, T. \& Yan, Y. Unusual defect physics in $\mathrm{CH}_{3} \mathrm{NH}_{3} \mathrm{Pbl}_{3}$ perovskite solar cell absorber. Appl. Phys. Lett. 104, 063903 (2014).

Pioneering theoretical work that revealed the excellent defect tolerance of $\mathrm{MAPbl}_{3}$, which led to high-efficiency solar cells.

56. Egger, D. A., Rappe, A. M. \& Kronik, L. Hybrid organic-inorganic perovskites on the move. Acc. Chem. Res. 49, 573-581 (2016)

57. Liu, S. et al. Ferroelectric domain wall induced band gap reduction and charge separation in organometal halide perovskites. J. Phys. Chem. Lett. 6, 693-699 (2015)

58. Fan, Z. et al. Ferroelectricity of $\mathrm{CH}_{3} \mathrm{NH}_{3} \mathrm{Pbl}$ perovskite. J. Phys. Chem. Lett. 6, 1155-116 (2015).

59. Frost, J. M. et al. Atomistic origins of highperformance in hybrid halide perovskite solar cells. Nano Lett. 14, 2584-2590 (2014)

60. Zheng, F., Takenaka, H., Wang, F., Koocher, N. Z. \& Rappe, A. M. First-principles calculation of the bulk photovoltaic effect in $\mathrm{CH}_{3} \mathrm{NH}_{3} \mathrm{Pbl}_{3}$ and $\mathrm{CH}_{3} \mathrm{NH}_{3} \mathrm{PbI}_{3-x} \mathrm{Cl}_{x}$. J. Phys. Chem. Lett. 6, 31-37 (2014).

61. Dang, Y. et al. Bulk crystal growth of hybrid perovskite material $\mathrm{CH}_{3} \mathrm{NH}_{3} \mathrm{Pbl}_{3}$. CrystEngComm 17, 665-670 (2015)

62. Aizu, K. Possible species of ferromagnetic ferroelectric, and ferroelastic crystals. Phys. Rev. B 2, 754-772 (1970)

63. Aizu, K. Possible species of "ferroelastic" crystals and of simultaneously ferroelectric and ferroelastic crystals. J. Phys. Soc. Jpn 27, 387-396 (1969).

64. Mosconi, E., Quarti, C., Ivanovska, T., Ruani, G. \& D Angelis, F. Structural and electronic properties of organo-halide lead perovskites: a combined IR-spectroscopy and $a b$ initio molecular dynamics investigation. Phys. Chem. Chem. Phys. 16, 16137-16144 (2014)

65. Chen, T. et al. Rotational dynamics of organic cations in the $\mathrm{CH}_{3} \mathrm{NH}_{3} \mathrm{Pbl}_{3}$ perovskite. Phys. Chem. Chem. Phys. 17, 31278-31286 (2015).

66. Leguy, A. M. et al. The dynamics of methylammonium ions in hybrid organic-inorganic perovskite solar cells. Nat. Commun. 67124 (2015).

67. Hermes, I. M. et al. Ferroelastic fingerprints in methylammonium lead iodide perovskite. J. Phys. Chem. C 120, 5724-5731 (2016).

68. Wei, J. et al. Hysteresis analysis based on the ferroelectric effect in hybrid perovskite solar cells. J. Phys. Chem. Lett. 5, 3937-3945 (2014).

69. Pintilie, L. \& Alexe, M. Ferroelectric-like hysteresis loop in nonferroelectric systems. Appl. Phys. Lett. 87, 112903 (2005)

70. Scott, J. Ferroelectrics go bananas. J. Phys. Condens. Matter 20, 021001 (2008).

71. Xiao, Z. et al. Giant switchable photovoltaic effect in organometal trihalide perovskite devices. Nat. Mater. 14, 193-198 (2015)

The first work that demonstrated the ion-migration effect and switchable photovoltaic effect in OIHPs.

72. Coll, M. et al. Polarization switching and lightenhanced piezoelectricity in lead halide perovskites. J. Phys. Chem. Lett. 6, 1408-1413 (2015)

73. Balke, N. et al. Local detection of activation energy for ionic transport in lithium cobalt oxide. Nano Lett. 12. 3399-3403 (2012)

74. Bark, C. et al. Switchable induced polarization in $\mathrm{LaAlO}_{3} / \mathrm{SrTiO}_{3}$ heterostructures. Nano Lett. 12 1765-1771 (2012).

75. Honig, M. et al. Local electrostatic imaging of striped domain order in $\mathrm{LaAlO}_{3} / \mathrm{SrTiO}_{3}$. Nat. Mater. 12 1112-1118 (2013).

76. Strelcov, E. et al. $\mathrm{CH}_{3} \mathrm{NH}_{3} \mathrm{Pbl}_{3}$ perovskites: ferroelasticity revealed. Sci. Adv. 3, e1602165 (2016).

77. Rothmann, M. U. et al. Direct observation of intrinsic twin domains in tetragonal $\mathrm{CH}_{3} \mathrm{NH}_{3} \mathrm{Pbl}_{3}$. Nat Commun. 8, 14547 (2017).

78. Liu, S., Zheng, F., Grinberg, I. \& Rappe, A. M. Photoferroelectric and photopiezoelectric properties of organometal halide perovskites. J. Phys. Chem. Lett. 7, 1460-1465 (2016)

79. Zhou, Y. et al. Giant photostriction in organicinorganic lead halide perovskites. Nat. Commun. 7 , 11193 (2016)

80. Mosconi, E. \& De Angelis, F. Mobile ions in organohalide perovskites: interplay of electronic structure and dynamics. ACS Energy Lett. 1, 182-188 (2016).

81. Fan, Z., Sun, K. \& Wang, J. Perovskites for photovoltaics: a combined review of organic-inorganic halide perovskites and ferroelectric oxide perovskites. J. Mater. Chem. A 3, 18809-18828 (2015).

82. Kulbak, M., Cahen, D. \& Hodes, G. How important is the organic part of lead halide perovskite photovoltaic cells? Efficient $\mathrm{CsPbBr}_{3}$ cells. J. Phys. Chem. Lett. 6 , 2452-2456 (2015)

83. Swarnkar, A. et al. Quantum dot-induced phase stabilization of $\alpha$-CsPbl perovskite for high-efficiency photovoltaics. Science 354, 92-95 (2016).
84. Zhu, H. et al. Organic cations might not be essential to the remarkable properties of band edge carriers in lead halide perovskites. Adv. Mater. 29, 1603072 (2016).

85. Zhu, H. et al. Screening in crystalline liquids protects energetic carriers in hybrid perovskites. Science 353, 1409-1413 (2016).

86. Guo, Z. et al. Long-range hot-carrier transport in hybrid perovskites visualized by ultrafast microscopy. Science 356, 59-62 (2017).

87. Zhang, C. et al. Charge recombination and band-edge shift in the dye-sensitized $\mathrm{Mg}^{2+}$-doped $\mathrm{TiO}_{2}$ solar cells. J. Phys. Chem. C 115, 16418-16424 (2011).

88. Stranks, S. D. et al. Electron-hole diffusion lengths exceeding 1 micrometer in an organometal trihalide perovskite absorber. Science 342, 341-344 (2013).

89. Gao, Y. et al. Surface doping of conjugated polymers by graphene oxide and its application for organic electronic devices. Adv. Mater. 23, 1903-1908 (2011).

90. Zhu, F. et al. The origin of higher open-circuit voltage in $\mathrm{Zn}$-doped $\mathrm{TiO}_{2}$ nanoparticle-based dye-sensitized solar cells. ChemPhysChem 13, 3731-3737 (2012).

91. Shi, Y. et al. Work function engineering of graphene electrode via chemical doping. ACS Nano 4, 2689-2694 (2010).

92. $\mathrm{Bi}, \mathrm{C}$. et al. Understanding the formation and evolution of interdiffusion grown organolead halide perovskite thin films by thermal annealing. J. Mater. Chem. A 2 , 18508-18514 (2014).

93. Etgar, L. et al. Mesoscopic $\mathrm{CH}_{3} \mathrm{NH}_{3} \mathrm{Pbl}_{3} / \mathrm{TiO}_{2}$ heterojunction solar cells. J. Am. Chem. Soc. 134 17396-17399 (2012)

94. Laban, W. A. \& Etgar, L. Depleted hole conductor-free lead halide iodide heterojunction solar cells. Energy Environ. Sci. 6, 3249-3253 (2013).

95. You, J. et al. Low-temperature solution-processed perovskite solar cells with high efficiency and flexibility. ACS Nano 8, 1674-1680 (2014).

96. Yuan, Y. et al. Anomalous photovoltaic effect in organic-inorganic hybrid perovskite solar cells. Sci. Adv. 3, e1602164 (2017)

The first observation of the anomalous photovoltaic effect in lateral OIHP solar cells, uncovering an abrupt band bending around grain boundaries caused by ion accumulation.

97. Pazos-Outón, L. M. et al. Photon recycling in lead iodide perovskite solar cells. Science 351, 1430-1433 (2016).

98. Fang, Y., Wei, H., Dong, Q. \& Huang, J. Quantification of re-absorption and re-emission processes to determine photon recycling efficiency in perovskite single crystals. Nat. Commun. 8, 14417 (2016).

99. Richter, J. M. et al. Enhancing photoluminescence yields in lead halide perovskites by photon recycling and light out-coupling. Nat. Commun. 7, 13941 (2016)

100. Xiao, Z., Meng, W., Wang, J., Mitzi, D. B. \& Yan, Y Searching for promising new perovskite-based photovoltaic absorbers: the importance of electronic dimensionality. Mater. Horiz. 4, 206-216 (2017).

101. Kim, Y. et al. Pure cubic-phase hybrid iodobismuthates $\mathrm{AgBi}_{2} \mathrm{I}_{7}$ for thin-film photovoltaics. Angew. Chem. Int. Ed. 55, 9586-9590 (2016).

102. Smith, I. C., Hoke, E. T., Solis-lbarra, D., McGehee, M. D. \& Karunadasa, H. I. A layered hybrid perovskite solar-cell absorber with enhanced moisture stability. Angew. Chem. Int. Ed. 126, 11414-11417 (2014).

103. Slavney, A. H. et al. Chemical approaches to addressing the instability and toxicity of lead-halide perovskite absorbers. Inorg. Chem. 56, 46-55 (2017).

104. De Wolf, S. et al. Organometallic halide perovskites sharp optical absorption edge and its relation to photovoltaic performance. J. Phys. Chem. Lett. 5 1035-1039 (2014).

105. Sadhanala, A. et al. Preparation of single-phase films of $\mathrm{CH}_{3} \mathrm{NH}_{3} \mathrm{~Pb}\left(\mathrm{l}_{1-x} \mathrm{Br}_{x}\right)_{3}$ with sharp optical band edges. J. Phys. Chem. Lett. 5, 2501-2505 (2014).

106. Samiee, M. et al. Defect density and dielectric constant in perovskite solar cells. Appl. Phys. Lett. 105, 153502 (2014).

107. McMeekin, D. P. et al. A mixed-cation lead mixedhalide perovskite absorber for tandem solar cells. Science 351, 151-155 (2016)

108. Wei, H. et al. Sensitive X-ray detectors made of methylammonium lead tribromide perovskite single crystals. Nat. Photonics 10, 333-339 (2016).

109. Steirer, K. X. et al. Defect tolerance in methylammonium lead triiodide perovskite. ACS Energy Lett. 1, 360-366 (2016). 
110. Mosconi, E., Meggiolaro, D., Snaith, H. J., Stranks, S. D. \& De Angelis, F. Light-induced annihilation of Frenkel defects in organo-lead halide perovskites. Energy Environ. Sci. 9, 3180-3187 (2016).

111. Zhang, W. et al. Photo-induced halide redistribution in organic-inorganic perovskite films. Nat. Commun. 7 11683 (2016).

112. Fang, Y., Dong, Q., Shao, Y., Yuan, Y. \& Huang, J. Highly narrowband perovskite single-crystal photodetectors enabled by surface-charge recombination. Nat. Photonics 9, 679-686 (2015)

113. Leblebici, S. Y. et al. Facet-dependent photovoltaic efficiency variations in single grains of hybrid halide perovskite. Nat. Energy 1, 16093 (2016).

114. Shao, Y., Xiao, Z., Bi, C., Yuan, Y. \& Huang, J. Origin and elimination of photocurrent hysteresis by fullerene passivation in $\mathrm{CH}_{3} \mathrm{NH}_{3} \mathrm{Pbl}_{3}$ planar heterojunction solar cells. Nat. Commun. 5, 5784 (2014).

This pioneering experimental work demonstrated that surface defects on OIHP can cause photocurrent hysteresis and that fullerene passivation can efficiently reduce photocurrent hysteresis.

115. Murali, B. et al. Surface restructuring of hybrid perovskite crystals. ACS Energy Lett. 1, 1119-1126 (2016).

116. Yang, Y. et al. Low surface recombination velocity in solution-grown $\mathrm{CH}_{3} \mathrm{NH}_{3} \mathrm{PbBr}_{3}$ perovskite single crystal. Nat. Commun. 6, 7961 (2015).

117. Zhang, F. et al. Film-through large perovskite grains formation via a combination of sequential thermal and solvent treatment. J. Mater. Chem. A 4, 8554-8561 (2016).

118. Nie, W. et al. High-efficiency solution-processed perovskite solar cells with millimeter-scale grains. Science 347, 522-525 (2015).

119. Chen, Q. et al. Under the spotlight: The organicinorganic hybrid halide perovskite for optoelectronic applications. Nano Today 10, 355-396 (2015)

120. Bi, C. et al. Non-wetting surface-driven high-aspectratio crystalline grain growth for efficient hybrid perovskite solar cells. Nat. Commun. 6, 7747 (2015).

121. de Quilettes, D. W. et al. Impact of microstructure on local carrier lifetime in perovskite solar cells. Science 348, 683-686 (2015).

122. Xing, G. et al. Low-temperature solution-processed wavelength-tunable perovskites for lasing. Nat. Mater. 13, 476-480 (2014)

123. Shi, D. et al. Low trap-state density and long carrier diffusion in organolead trihalide perovskite single crystals. Science 347, 519-522 (2015).

124. Saidaminov, M. I. et al. High-quality bulk hybrid perovskite single crystals within minutes by inverse temperature crystallization. Nat. Commun. 6, 7586 (2015).

125. Bi, Y. et al. Charge carrier lifetimes exceeding $15 \mu \mathrm{s}$ in methylammonium lead iodide single crystals. J. Phys. Chem. Lett. 7, 923-928 (2016).

126. Leijtens, T. et al. Carrier trapping and recombination: the role of defect physics in enhancing the open circuit voltage of metal halide perovskite solar cells. Energy Environ. Sci. 9, 3472-3481 (2016).
127. Baeg, K. J., Binda, M., Natali, D., Caironi, M. \& Noh, Y. Y. Organic light detectors: photodiodes and phototransistors. Adv. Mater. 25, 4267-4295 (2013).

128. Guo, F. et al. A nanocomposite ultraviolet photodetector based on interfacial trap-controlled charge injection. Nat. Nanotechnol. 7, 798-802 (2012).

129. Yuan, Y. \& Huang, J. Ultrahigh gain, low noise, ultraviolet photodetectors with highly aligned organic crystals. Adv. Opt. Mater. 4, 264-270 (2016).

130. Shao, Y., Yuan, Y. \& Huang, J. Correlation of energy disorder and open-circuit voltage in hybrid perovskite solar cells. Nat. Energy 1, 15001 (2016).

131. Ihly, R. et al. Efficient charge extraction and slow recombination in organic-inorganic perovskites capped with semiconducting single-walled carbon nanotubes. Energy Environ. Sci. 9, 1439-1449 (2016).

132. Schulz, P. et al. Charge transfer dynamics between carbon nanotubes and hybrid organic metal halide perovskite films. J. Phys. Chem. Lett. 7, 418-425 (2016).

133. Wang, Q., Liu, X., Shao, Y., Gao, Y. \& Huang, J. Qualification of $\mathrm{p}$ and $\mathrm{n}$ self-doping in $\mathrm{CH}_{3} \mathrm{NH}_{3} \mathrm{Pbl}_{3}$ Films. Appl. Phys. Lett. 105, 163508 (2014).

134. Wetzelaer, G. J. A. et al. Trap-assisted non-radiative recombination in organic-inorganic perovskite solar cells. Adv. Mater. 27, 1837-1841 (2015).

135. Adinolfi, V. et al. The in-gap electronic state spectrum of methylammonium lead iodide single-crystal perovskites. Adv. Mater. 28, 3406-3410 (2016).

136. Liu, P. et al. Interfacial electronic structure at the $\mathrm{CH}_{3} \mathrm{NH}_{3} \mathrm{Pbl}_{3} / \mathrm{MoO}_{x}$ interface. Appl. Phys. Lett. 106, 193903 (2015)

137. Wu, X. et al. Trap states in lead iodide perovskites J. Am. Chem. Soc. 137, 2089-2096 (2015)

138. Chae, J., Dong, Q., Huang, J. \& Centrone, A. Chloride incorporation process in $\mathrm{CH}_{3} \mathrm{NH}_{3} \mathrm{Pbl}_{3-x} \mathrm{Cl}_{x}$ perovskites via nanoscale bandgap maps. Nano Lett. 15 8114-8121 (2015).

139. Jiang, M. et al. Enhancing the performance of planar organo-lead halide perovskite solar cells by using a mixed halide source. J. Mater. Chem. A 3, 963-967 (2015).

140. Brenner, T. M., Egger, D. A., Kronik, L., Hodes, G. \& Cahen, D. Hybrid organic-inorganic perovskites: lowcost semiconductors with intriguing charge-transport properties. Nat. Rev. Mater. 1, 15007 (2016).

141. Yuan, Y., Wang, Q. \& Huang, J. in Organic-Inorganic Halide Perovskite Photovoltaics: From Fundamentals to Device Architectures (eds Park, N.-G., Grätzel, M. \& Miyasaka, T.) 137-162 (Springer, 2016)

142. Yuan, Y. \& Huang, J. Ion migration in organometal trihalide perovskite and its impact on photovoltaic efficiency and stability. Acc. Chem. Res. 49, 286-293 (2016).

143. Yuan, Y., Xiao, Z., Yang, B. \& Huang, J. Arising applications of ferroelectric materials in photovoltaic devices. J. Mater. Chem. A 2, 6027-6041 (2014).

144. Yuan, Y. et al. Photovoltaic switching mechanism in lateral structure hybrid perovskite solar cells. Adv. Energy Mater. 5, 1500615 (2015).
145. Deng, Y., Xiao, Z. \& Huang, J. Light induced self-poling effect in organometal trihalide perovskite solar cells for increased device efficiency and stability. Adv. Energy Mater. 5, 1500721 (2015).

146. Zou, Y. \& Holmes, R. J. Temperature-dependent bias poling and hysteresis in planar organo-metal halide perovskite photovoltaic cells. Adv. Energy Mater. 6 1501994 (2016).

147. Nie, W. et al. Light-activated photocurrent degradation and self-healing in perovskite solar cells. Nat. Commun. 7, 11574 (2016).

148. Huang, F. et al. Fatigue behavior of planar $\mathrm{CH}_{3} \mathrm{NH}_{3} \mathrm{Pbl}_{3}$ perovskite solar cells revealed by light on/off diurnal cycling. Nano Energy 27, 509-514 (2016).

149. Xing, J. et al. Ultrafast ion migration in hybrid perovskite polycrystalline thin films under light and suppression in single crystals. Phys. Chem. Chem. Phys. 18, 30484-30490 (2016).

150. Yuan, Y. et al. Electric field driven reversible conversion between methylammonium lead triiodide perovskites and lead iodide at elevated temperature. Adv. Energy Mater. 6, 1501803 (2015).

151. Zhao, Y. et al. A polymer scaffold for self-healing perovskite solar cells. Nat. Commun. 7, 10228 (2016).

152. Shockley, W. \& Queisser, H. J. Detailed balance limit of efficiency of $\mathrm{p}-\mathrm{n}$ junction solar cells. J. Appl. Phys. 32, 510-519 (1961)

153. Kirchartz, T., Rau, U., Kurth, M., Mattheis, J. \& Werner, J. Comparative study of electroluminescence from $\mathrm{Cu}(\mathrm{In}, \mathrm{Ga}) \mathrm{Se}_{2}$ and Si solar cells. Thin Solid Films 515, 6238-6242 (2007).

154. Tvingstedt, K. et al. Radiative efficiency of lead iodide based perovskite solar cells. Sci. Rep. 4, 6071 (2014).

155. Yao, J et al Quantifying losses in open-circuit voltage in solution-processable solar cells. Phys. Rev. Appl. 4 , 014020 (2015).

156. Bi, D. et al. Efficient luminescent solar cells based on tailored mixed-cation perovskites. Sci. Adv. 2, e1501170 (2016)

\section{Acknowledgements}

The authors are grateful for the financial support from the Office of Naval Research (ONR) (Grant No. N00014-15-1-2713), Air Force Office of Scientific Research (AFOSR) (Grant No. A9550-16-1-0299) and National Science Foundation under awards of OIA-1538893, ECCS-1252623 and DMR-1505535. Y. Yuan also thanks the National Natural Science Foundation of China (Grant No. 51673218) for financial support.

\section{Competing interests statement}

The authors declare no competing interests.

\section{Publisher's note}

Springer Nature remains neutral with regard to jurisdictional claims in published maps and institutional affiliations.

\section{How to cite this article}

Huang, J., Yuan, Y., Shao, Y. \& Yan, Y. Understanding the physical properties of hybrid perovskites for photovoltaic applications. Nat. Rev. Mater. 2, 17042 (2017). 